\title{
Temporal dynamics of Wnt-dependent transcriptome reveal an oncogenic Wnt/MYC/ribosome axis
}

\author{
Babita Madan, ${ }^{1}$ Nathan Harmston, ${ }^{1,2}$ Gahyathiri Nallan, ${ }^{1}$ Alex Montoya, ${ }^{3}$ Peter Faull, ${ }^{3}$ Enrico Petretto, ${ }^{2,3}$ and David M. Virshup ${ }^{1,4}$ \\ 'Programme in Cancer and Stem Cell Biology, Duke-NUS Medical School, Singapore. ${ }^{2}$ Centre for Computational Biology and Programme in Cardiovascular and Metabolic Disorders, Duke-NUS Medical School, \\ Singapore. ${ }^{3}$ MRC London Institute of Medical Sciences, Imperial College London, Hammersmith Campus, London, United Kingdom. ${ }^{4}$ Department of Pediatrics, Duke University School of Medicine, Durham, \\ North Carolina, USA.
}

\begin{abstract}
Activating mutations in the Wnt pathway drive a variety of cancers, but the specific targets and pathways activated by Wnt ligands are not fully understood. To bridge this knowledge gap, we performed a comprehensive time-course analysis of Wnt-dependent signaling pathways in an orthotopic model of Wnt-addicted pancreatic cancer, using a porcupine (PORCN) inhibitor currently in clinical trials, and validated key results in additional Wnt-addicted models. The temporal analysis of the drug-perturbed transcriptome demonstrated direct and indirect regulation of more than 3,500 Wnt-activated genes (23\% of the transcriptome). Regulation was both via Wnt/ $\beta$-catenin and through the modulation of protein abundance of important transcription factors, including MYC, via Wnt-dependent stabilization of proteins (Wnt/STOP). Our study identifies a central role of Wnt/ $\beta$-catenin and Wnt/STOP signaling in controlling ribosome biogenesis, a key driver of cancer proliferation.
\end{abstract}

\section{Introduction}

Wnts are a family of 19 secreted proteins that play key roles in cell proliferation, cell-cell communication, and cell differentiation and that are essential during embryonic development and in adult tissue homeostasis (1). The binding of Wnts to their receptors and coreceptors results in the regulation of multiple downstream signaling pathways (2). Our knowledge of the specific events and signals regulated by Wnts derives from a variety of genetic, molecular, and biochemical approaches that have generated a rich map of these downstream pathways $(3,4)$.

The Wnt/ $\beta$-catenin pathway, also known as canonical WNT signaling, has been intensively studied. In the presence of Wnts, $\beta$-catenin is stabilized and translocates to the nucleus, where it drives expression of target genes in a context-specific manner via binding to TCF7L2 and other factors $(3,5,6)$. In addition to the Wnt/ $\beta$-catenin pathway, Wnts regulate signaling through diverse $\beta$-catenin-independent noncanonical pathways, such as the planar cell polarity (PCP) pathway and the Wnt-dependent stabilization of proteins (Wnt/STOP) pathway, which are less well characterized (7-11).

While the downstream mutations that stabilize $\beta$-catenin (e.g., in the adenomatous polyposis coli $[A P C]$ gene) clearly cause human cancers, genetic lesions that cause Wnt overexpression have not been found (12). A subset of mutations that block Wnt receptor internalization and confer dependency on Wnt ligands have been identified in a range of carcinomas. These include loss-

Authorship note: $\mathrm{BM}$ and $\mathrm{NH}$ contributed equally to this work.

Conflict of interest: BM and DMV have a financial interest in ETC-159 (patent W02014/175832).

License: Copyright 2018, American Society for Clinical Investigation.

Submitted: May 22, 2018; Accepted: October 2, 2018.

Reference information: J Clin Invest. 2018;128(12):5620-5633.

https://doi.org/10.1172/JCI122383. of-function mutations in RNF43, an E3-ligase, and translocations leading to increased R-spondin levels (13-15). RNF43 mutations (16) and translocations involving RSPO2 and RSPO3 are found in $7 \%$ of pancreatic adenocarcinoma (PDAC) and $10 \%$ of colorectal cancers, respectively (15). Cancers with RNF43 or RSPO3 mutations have a markedly increased abundance of Frizzled receptors on the cell surface and are uniquely Wnt addicted $(17,18)$.

Wnts are palmitoleated by a membrane-bound O-acyltransferase, porcupine (PORCN). This modification is essential for binding to chaperone WLS and Frizzled receptors and is therefore required for the activity of all Wnts (19). Pharmacological PORCN inhibitors, such as ETC-159 and LGK-974, have progressed to phase I clinical trials due to their efficacy in preclinical models of RNF43-mutant pancreatic and RSPO3-translocated colorectal cancers $(13,18,20)$. The recent development of these PORCN inhibitors that block all Wnt secretion provides an opportunity to investigate how Wnt-regulated genes change over time following withdrawal of signaling $(18,21-25)$. In order to provide the most relevant data, it is important to use the most predictive preclinical models. A large body of literature demonstrates that cancers in vivo behave very differently than cancers in tissue culture (26). These differences have led to the development of orthotopic xenograft models and patient-derived xenografts that better reflect the behavior of Wnt-addicted cancers in a complex cancer-host environment (27).

Here, we investigated the temporal impact of acute withdrawal of Wnt ligands on the transcriptome of a Wnt-addicted human pancreatic cancer in an orthotopic mouse model. The timeseries analysis identified direct and indirect Wnt targets based on their dynamics, distinguishing immediate-early, early, and lateresponse genes. We identified and confirmed an important role of the Wnt/STOP pathway in regulating tumor growth and disentangled the WNT versus MYC dependencies. This comprehensive time-course analysis in an in vivo Wnt-addicted cancer provides 


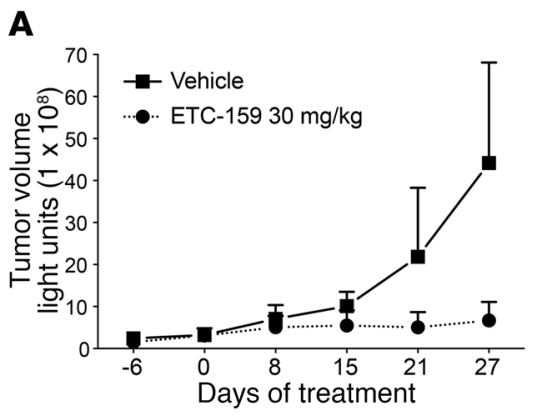

B

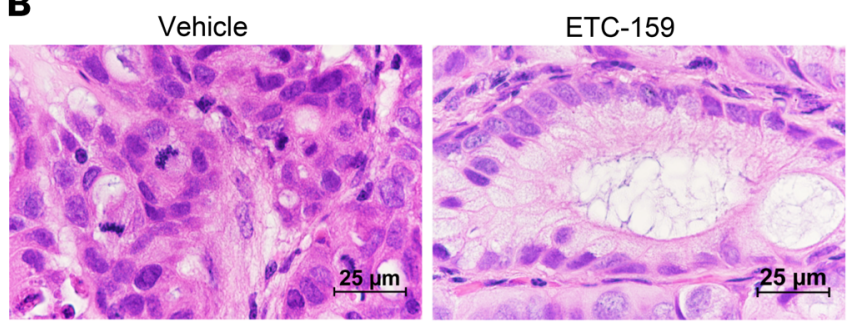

C

Orthotopic injection

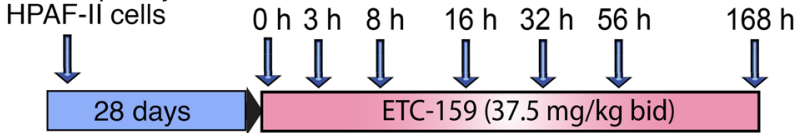

D

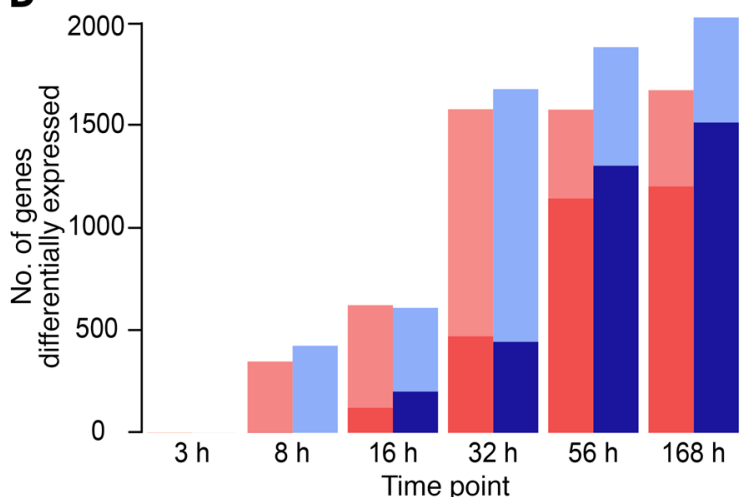

Upregulated Also upregulated at previous timepoint Downregulated Also downregulated at previous timepoin

Figure 1. PORCN inhibition remodels the morphology and transcriptome of RNF43 mutant pancreatic cancer. (A) ETC-159 treatment prevents the growth of orthotopic HPAF-II xenografts. HPAF-II cells $\left(10^{6}\right)$ were injected into the tail of the mouse pancreas. Following establishment of tumors ( $\sim 3$ weeks), the mice were treated daily with $30 \mathrm{mg} / \mathrm{kg}$ ETC-159. Tumor growth was monitored by measuring luciferase activity. Data represent mean \pm SD. $n=8$ /group. (B) Inhibition of Wnt signaling promotes histological changes in HPAF-II xenografts. H\&E-stained images of HPAF-II xenografts treated with ETC-159 for 28 days. (C) Schematic representation of the experimental plan. HPAF-II cells $\left(10^{6}\right)$ were injected into the tail of the mouse pancreas. Following establishment of tumors ( 28 days), the mice were treated twice daily with $37.5 \mathrm{mg} / \mathrm{kg}$ doses of ETC-159. Tumors were harvested at the indicated time points. (D) ETC-159 treatment leads to widespread changes in the transcriptome. Total number of genes whose expression changes after PORCN inhibition over time compared with at 0 hours. Genes whose expression was up- or downregulated at the previous time point are also indicated (absolute fold change $>1.5$, FDR $<10 \%$ ). insights into the central role of Wnt/ $\beta$-catenin and Wnt/STOP signaling in the regulation of the ribosome biogenesis pathway.

\section{Results}

Time-dependent global transcriptional changes follow PORCN inhibition in a Wnt-addicted pancreatic cancer model. We aimed to identify the genes and biological processes that are directly or indirectly regulated by Wnt ligands in a Wnt-addicted cancer in vivo. To mirror the tumor microenvironment and recapitulate tumor-stromal interactions, we established an orthotopic mouse model using a highly WNT-dependent HPAF-II cell line with RNF43-inactivating mutation $(13,18)$. HPAF-II cells stably expressing firefly luciferase were injected into the mouse pancreas, and tumor growth was monitored by measuring light output. As expected, ETC-159 significantly inhibited the growth of HPAF-II orthotopic xenografts (Figure 1A) and led to pronounced histomorphological changes (Figure 1B) (18). Tumors from the control group were characterized by the presence of neoplastic cells with poorly defined acini and cell boundaries. Treatment with ETC-159 induced changes in cellular organization and, by 7 days, the tissue appeared more differentiated, with a decreased nuclear cytoplasmic ratio and diminished anisocytosis and anisokaryosis. There were groups of slender elongated cells that often palisaded with uniform cellular and nuclear polarity, had distinct cell borders, and formed acini. The acinar lumens had abundant mucus, as seen by Alcian blue staining (Supplemental Figure $1 \mathrm{~A}$; supplemental material available online with this article; https:// doi.org/10.1172/JCI122383DS1).

To identify immediate-early, early, and late responses to Wnt inhibition, mice with established HPAF-II orthotopic xenografts were treated with ETC-159 and tumors were collected at 3, 8, 16, 32, and 56 hours and at 7 days after treatment (Figure 1C). Comprehensive gene expression analysis was performed using RNA-Seq of 4 to 7 independent tumors at each time point (Supplemental Figure 1A). Inhibition of WNT signaling led to a marked change in the transcriptome, with the expression of 11,673 genes ( $75 \%$ of all expressed genes) changing over time (FDR $<10 \%)$ (Supplemental Table 1). Expression of 773 genes changed as early as 8 hours after the first dose of ETC-159. After 56 hours, 1,578 and 1,883 genes were upregulated or downregulated, respectively (FDR $<10 \%$, absolute fold-change $>1.5$ ) (Figure 1D and Supplemental Figure 1, B and C). The majority of genes that exhibited significant differences at 56 hours were also differentially expressed at 7 days, suggesting that the effect of Wnt inhibition is primarily established within 3 days.

To better understand how the withdrawal of Wnt signaling affected gene expression over time, we performed time-series clustering (28) of differentially expressed genes. Genes with significant changes in response to treatment were grouped into 64 clusters, with each cluster consisting of genes exhibiting similar dynamic responses following PORCN inhibition (Supplemental Figure 1D and Supplemental Table 2). Further analysis of these clusters to identify consistent global patterns of transcriptional response identified 2 major robust patterns (Supplemental Figure1, $\mathrm{E}-\mathrm{F}$ ), a supercluster comprising genes consistently downregulated (Wnt-activated genes) and a supercluster containing genes consistently upregulated following PORCN inhibition (Wnt-repressed genes) (Figure 2A). Here, we focus on Wnt-activated genes, and 
A

Orthotopic injection

HPAF-II cells

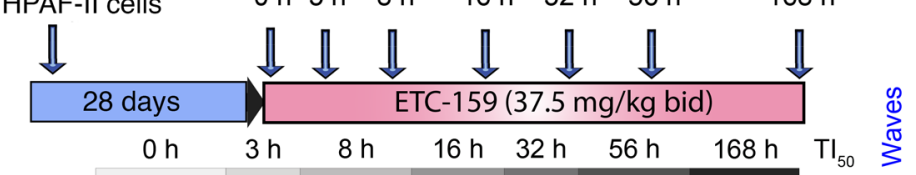

$\mathrm{C} 20$ -

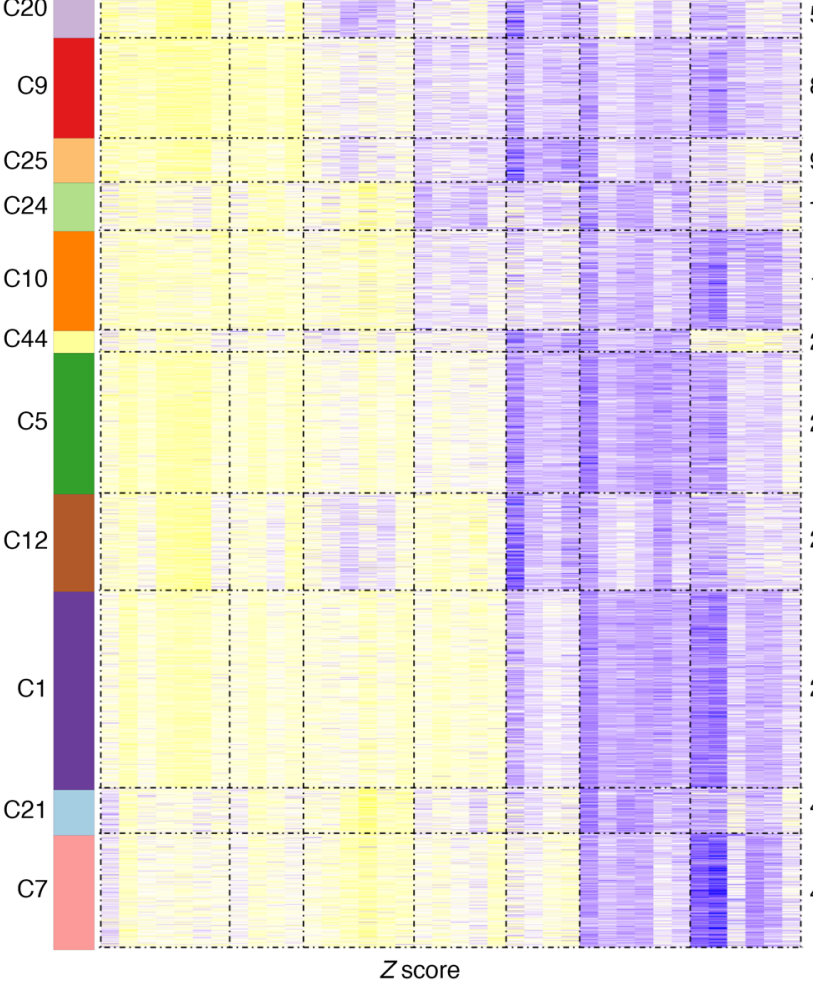

$\left.\begin{array}{l}5.3 \\ 8.5 \\ 9.8\end{array}\right]-1$

$\left.{ }_{15.1}^{12.9}\right]^{-2}$

20.3

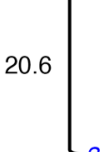

22.9

26.5

$\left.{ }_{46.6}^{40.8}\right]^{-4}$

B

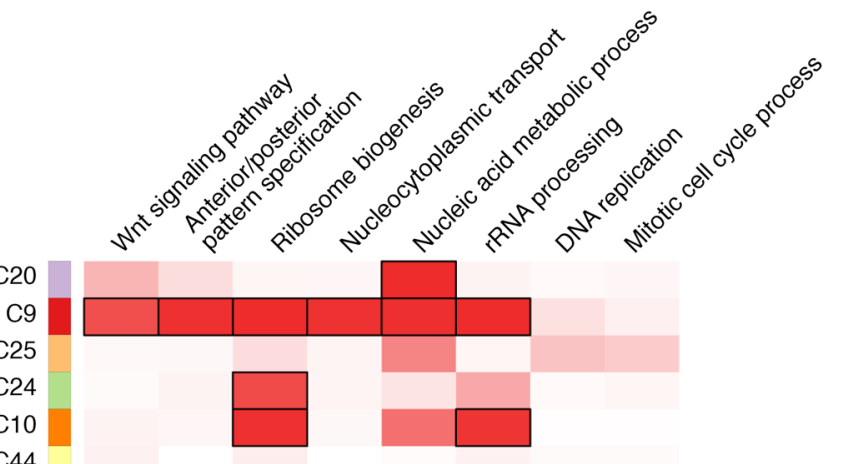

$\mathrm{C} 44$

C5 12

C1

C21

C7

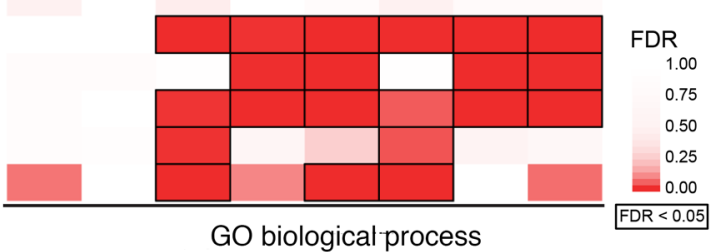

$\begin{array}{lllllll}-3 & -2 & -1 & 0 & 1 & 2 & 3\end{array}$
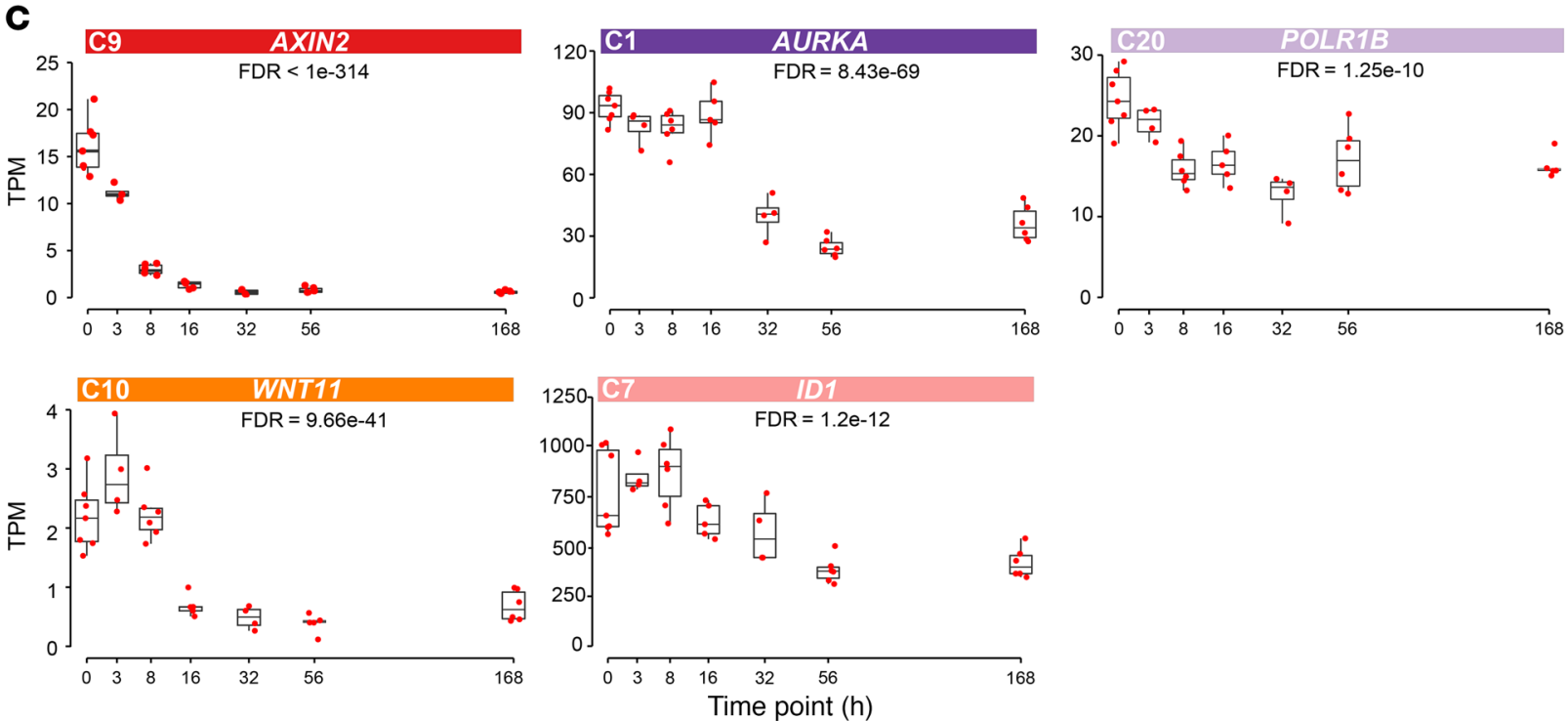

Figure 2. Time-series clustering reveals distinct dynamics of Wnt-regulated genes and enrichment for cell cycle and ribosome biogenesis. (A) Timeseries clustering of Wnt-activated genes reveals distinct patterns of response to PORCN inhibition. Genes differentially expressed over time (FDR $<10 \%)$ in response to PORCN inhibition were clustered by expression pattern using GP Clust. The cluster number (C) and $\mathrm{Tl}_{50}$ for each cluster are indicated. (B) $\mathrm{CO}$ Biological Process enrichments of each cluster of Wnt-activated genes (hypergeometric test). Enrichment of Wnt-activated genes highlights processes, including Wnt signaling, ribosome biogenesis, and the cell cycle. (C) Illustration of the various time courses of gene expression following PORCN inhibition. Representative Wnt-activated genes from selected clusters are shown. TPM, transcripts per million reads. (D) Well-established Wnt/ $\beta$-catenin target genes change with distinct dynamics following PORCN inhibition. 
we provide the analysis of Wnt ligand-regulated genes in Supplemental Tables 1 and 2.

Analysis of Wnt-activated genes. The Wnt-activated genes supercluster contained 11 clusters with distinct dynamics, consisting of 3,549 genes ( $23 \%$ of the transcriptome). For each of these clusters, we calculated the time to $50 \%$ inhibition $\left(\mathrm{TI}_{50}\right)$ based on its mean profile. We operatively classified these clusters into 4 waves, with $\mathrm{TI}_{50}$ ranging from 5.3 to 46.6 hours (Figure 2A) (first wave, 5.3-9.8 hours; second, 12.9-15.1; third, 20.3-26.5; fourth, 40.8-46.6). Well-established Wnt target genes had distinct time courses and were present throughout the first 3 waves, with $\mathrm{TI}_{50}$ ranging from 5.3 to 26.5 hours. For example, cluster $9\left(\mathrm{TI}_{50}=8.5\right.$ hours $)$ included well-known $\beta$-catenin targets (e.g., AXIN2, NKD1, RNF43, BMP4, and $L G R 5)$ and was significantly enriched for pathways and processes relating to Wnt signaling and development (Figure 2B). $\mathrm{C} 5$ and $\mathrm{C} 12$ in the third wave $\left(\mathrm{TI}_{50}, 20.6\right.$ and 22.9) similarly contained known Wnt target genes, e.g., NOTUM (29). The complex dynamics and broad range of response times of the various $\beta$-catenin targets most likely relates to the cell-type-specific context of the coregulatory elements of these genes and the stability of the specific mRNAs.

Interestingly, we observed that the early changing clusters, such as C9, contained genes that are not known to be direct $\beta$-catenin target genes (Supplemental Figure 2) and thus may be $\beta$-catenin independent and rely on mechanisms such as Wnt/ STOP. These included well-studied regulators of ribosome biogenesis (e.g., NPM1, DKC1, NOL6, RRS1) and nucleocytoplasmic transport (e.g., XPO5, NUP37) (30). Other smaller, rapidly responding clusters in the first and second waves similarly contained genes not known to be $\beta$-catenin target genes. These clusters were also enriched for processes associated with ribosome biogenesis (e.g., C20: POLR1A, POLR1B; C25: NOP14, RRP9). The slowest responding genes, found in the fourth wave clusters

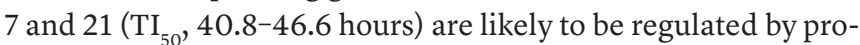
cesses downstream of initial Wnt signaling events and were also enriched for processes relating to ribosome biogenesis (Figure 2, $\mathrm{A}$ and $\mathrm{B}$ ). In addition to ribosome biogenesis, there was a broad enrichment throughout the clusters for genes involved in nucleic acid metabolism and cell cycle, especially in the third wave, C1, C5, and C12 (Figure 2B). Selected examples of Wnt-activated genes with differences in their pattern of response to Wnt inhibition are depicted in Figure 2, C and D.

Our data set provides a comprehensive resource of genes whose expression is highly dependent on Wnt signaling in vivo (Supplemental Table 1). As several of the early changing genes are not known to be direct targets of $\beta$-catenin, this analysis identified genes that may depend on additional pathways, such as Wnt/ STOP. Importantly, the data set highlights that, in addition to its recognized role in cell-cycle regulation, an early consequence of blocking Wnt signaling is the downregulation of genes involved in ribosome biogenesis and its associated processes.

A common core of Wht-regulated gene expression changes are more robust in orthotopic xenografts. To determine whether the gene expression changes seen in the HPAF-II pancreatic cancer were generalizable to other Wnt-addicted cancers, we compared our data to our previously published data set of a Wnt-addicted
RSPO3-translocation patient-derived colorectal cancer xenograft (CRC PDX) (Supplemental Table 3 and Supplemental Figure 3) (18). The strength of the correlation between the expression changes induced by PORCN inhibition at 56 hours in the 2 experimental systems $\left(r^{2}=0.36\right.$, Figure $\left.3 \mathrm{~A}\right)$ indicated that, regardless of the upstream mutation and tissue of origin, the downstream effect of Wnt inhibition on tumor gene expression was similar. In keeping with the central role of Wnts in regulating the differentially expressed genes, the majority of $W n t$-activated genes (62\%, FDR $<10 \%$, absolute fold-change $>1.5$ ) were also downregulated at 56 hours in the CRC PDX (Figure 3B). Within C9, 69\% of the genes expressed in both systems were downregulated in CRC PDX, suggesting that these genes may be direct targets of WNT signaling in both of these tumor models. Similar changes were seen in an orthotopic ASPC-1 tumor treated at a single time point (Supplemental Figure 3F). This highlights that the core processes and mechanisms responsible for the Wnt-activated genes are shared between the CRC PDX and pancreatic cancer orthotopic xenografts. Notably, the overlapping set of Wnt-activated genes was enriched for genes involved in cell-cycle regulation and ribosome biogenesis (Supplemental Figure 3A), again suggesting the centrality of these pathways in Wnt-addicted cancers.

To determine whether the orthotopic model was a more robust Wnt target discovery system, we compared the effect of ETC-159 on HPAF-II cells in vitro or as a subcutaneous xenograft. The differential expression analysis identified 4,409 genes whose response to ETC-159 was significantly different (interaction test, FDR $<10 \%$ ) between models (see Supplemental Information for details) (Supplemental Table 4 and Figure 3, C-F, and Supplemental Figure 3, A-E). These genes, e.g., AURKA, cyclin E1, and $C D K N 2 B$, were enriched for processes, including cell cycle and ribosome biogenesis (Figure 3F and Supplemental Figure 3B). In addition to cell-cycle-associated genes, we identified several other genes that did not respond to Wnt inhibition in vitro, but behaved as WNT targets in vivo (i.e., EPHB3 and TGFBI; Supplemental Figure 3C). Looking only at genes decreasing more than 1.5 -fold at 56 hours, we would have missed 817/1867 (44\%) genes using a subcutaneous or 939/1867 (50\%) using an in vitro model. Thus, the overall response to Wnt inhibition was reduced in the subcutaneous model and further blunted in vitro.

The much less pronounced effect of ETC-159 on the expression of cell-cycle genes in cell culture is consistent with our previous observations that PORCN inhibitors do not inhibit the growth of Wnt-addicted cancer cells in short-term $2 \mathrm{D}$ cell culture (20). These data demonstrate that Wnt target genes are highly dependent on the tissue-specific microenvironment, highlighting the value of using an orthotopic model to identify Wnt-regulated genes.

PORCN inhibition leads to early downregulation of MYC and its targets. The time-series clustering analysis (Figure 2A) identified sets of genes (clusters) having similar dynamics of response to PORCN inhibition, suggesting that each cluster may be regulated by distinct mechanisms. To investigate the differences in the transcriptional regulation of these genes, we performed a transcription factor-binding site (TFBS) motif analysis on the promoters of the Wnt-activated genes (Figure 4A).

Unexpectedly, the promoters of genes downregulated immediately following Wnt withdrawal (e.g. C9, $\mathrm{TI}_{50}=8.5$ hours) did 

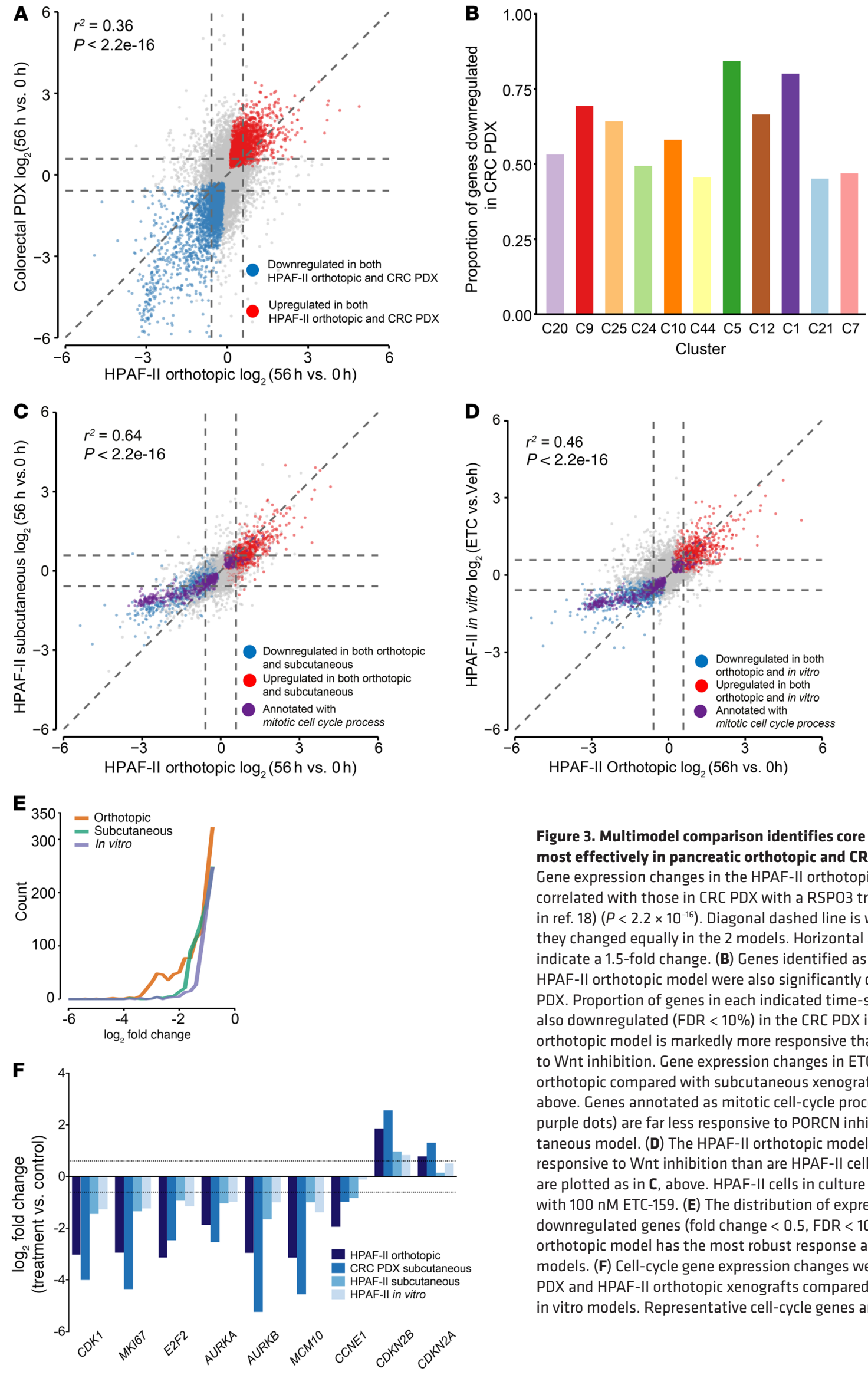

Figure 3. Multimodel comparison identifies core Wnt-activated genes most effectively in pancreatic orthotopic and CRC PDX models. (A) Gene expression changes in the HPAF-II orthotopic xenografts are highly correlated with those in CRC PDX with a RSPO3 translocation (described in ref. 18) $\left(P<2.2 \times 10^{-16}\right)$. Diagonal dashed line is where genes would fall if they changed equally in the 2 models. Horizontal and vertical dashed lines indicate a 1.5-fold change. (B) Genes identified as Wnt activated in the HPAF-II orthotopic model were also significantly downregulated in the CRC PDX. Proportion of genes in each indicated time-series cluster that were also downregulated (FDR $<10 \%$ ) in the CRC PDX is shown. (C) The HPAF-II orthotopic model is markedly more responsive than a subcutaneous model to Wnt inhibition. Gene expression changes in ETC-159-treated HPAF-II orthotopic compared with subcutaneous xenografts are plotted as in $\mathbf{A}$, above. Genes annotated as mitotic cell-cycle processes (GO BP1903047, purple dots) are far less responsive to PORCN inhibition in the subcutaneous model. (D) The HPAF-II orthotopic model is dramatically more responsive to Wnt inhibition than are HPAF-II cells cultured in vitro; data are plotted as in C, above. HPAF-II cells in culture were treated for 48 hours with $100 \mathrm{nM}$ ETC-159. (E) The distribution of expression changes for the downregulated genes (fold change $<0.5$, FDR $<10 \%$ ) illustrates that the orthotopic model has the most robust response across 3 different HPAF-II models. (F) Cell-cycle gene expression changes were more robust in CRC PDX and HPAF-II orthotopic xenografts compared with subcutaneous and in vitro models. Representative cell-cycle genes are shown. 
A

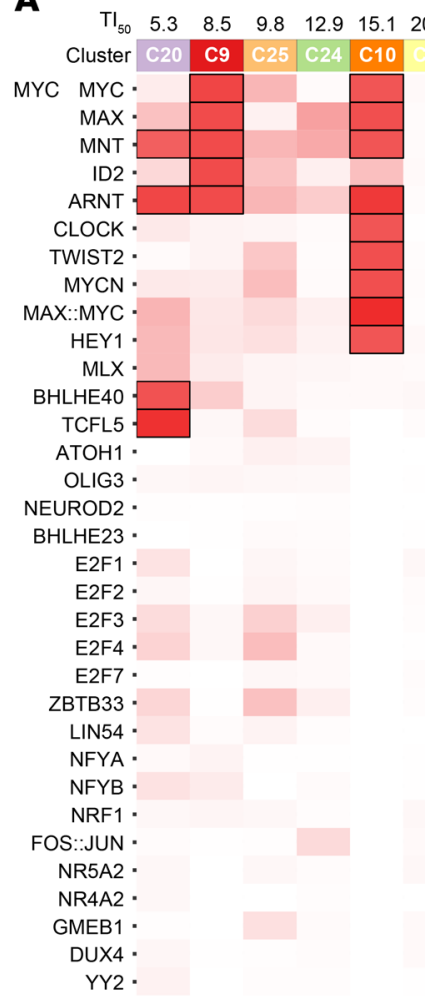

B

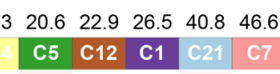

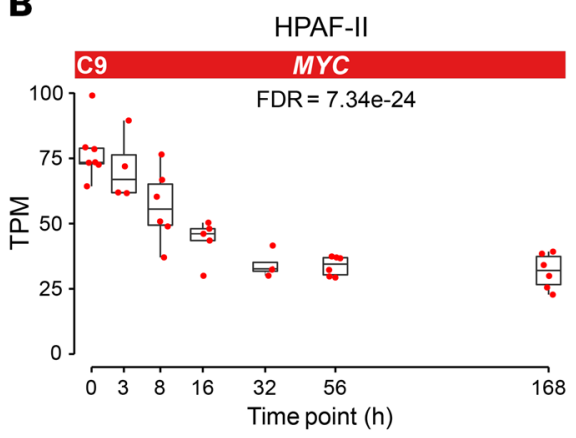

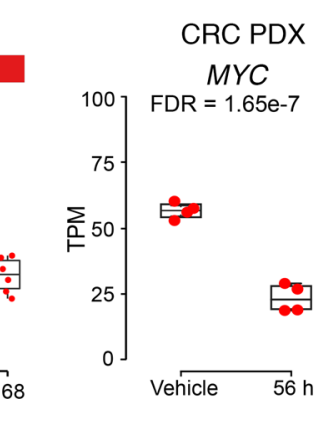

C
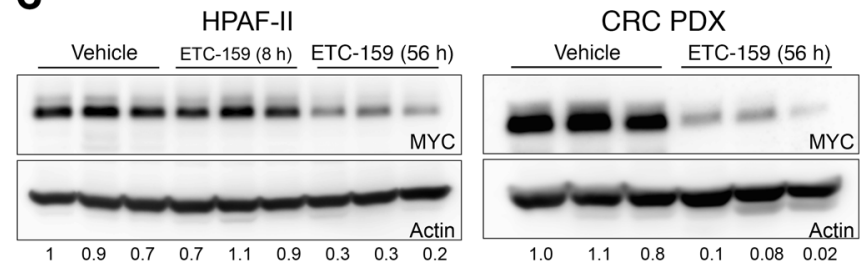

FDR

100

0.75

0.25

0.00

FDR $<5 \%$

Figure 4. The waves of Wnt-activated genes are associated with distinct sets of TFBSs with enrichment for E-boxes in the early responding clusters. (A) Clusters of Wnt-activated genes are enriched (FDR $<5 \%$ ) for distinct TFBS motifs (hypergeometric test). Promoters of genes in each cluster were scanned for motifs present in JASPAR 2016 using FIMO (53), and enrichment for each cluster was calculated. Promoters of genes in the early downregulated clusters are enriched for canonical E-boxes. (B) MYC gene expression is partially inhibited by ETC-159 treatment of HPAF-II orthotopic tumors and a CRC PDX (18). (C) MYC protein abundance is reduced in both HPAF-II orthotopic xenografts and CRC PDX following 56 hours treatment with ETC-159. The ratio of MYC protein compared with $\beta$-actin protein abundance for each lane is indicated.

not show significant enrichment for TCF7L2/LEF1-binding sites $(P=0.21)$. The majority of TCF7L2-binding events are found to be intergenic rather than promoter associated (Supplemental Figure 4) (31). The promoters of genes in the most rapidly responding clusters (i.e., C9, C10, C20, C24, and C25; $\mathrm{TI}_{50}<20$ hours) were rather significantly enriched for canonical E-box motifs, bound by transcription factors, including MYC, HEY1, CLOCK, and ID2 (Figure $4 \mathrm{~A})$. The genes in clusters that responded later $\left(\mathrm{TI}_{50}>20\right.$ hours) were enriched for E2F, NRF, and NFYB binding sites (32).

The enrichment of E-box-binding motifs occurred in some early responding Wnt-activated genes (e.g., C2O, C9) whose expression fell even before MYC mRNA decreased, suggesting additional levels of regulation (Figure 4, A and B). Upon PORCN inhibition, MYC mRNA responded as a direct WNT target gene with an early and sustained decrease (C9), albeit only to approximately $50 \%$ of its initial mRNA abundance (Figure $4 \mathrm{~B}$ ). This is consistent with the well-established role of $\beta$-catenin signaling in the regulation of MYC expression (33). In addition to being a transcriptional target of Wnt signaling, MYC protein abundance can be directly regulated by GSK3 by phosphorylating it at threonine 58 , priming it for ubiquitylation and proteasomal degradation $(8,9$, 34,35). As Wnt signaling inhibits AXIN-associated GSK3, blocking Wnt signaling increases the activity of GSK3 and promotes MYC degradation. Indeed, we observed a more pronounced change in MYC protein (3.3- to 14.5-fold reduction) than in MYC mRNA (2.2- to 2.0-fold reduction) in both HPAF-II orthotopic tumors and the CRC PDX models 56 hours after PORCN inhibitor treatment (Figure 4, B and C). These results suggest that the Wnt-dependent decrease in $M Y C$ transcripts was coupled with posttranscriptional regulation of MYC protein abundance, i.e., a Wnt/STOP effect in Wnt-addicted tumors.

Wnt signaling regulates MYC via WNT/STOP and the Wnt/ $\beta$ catenin pathway. To assess the relative contributions of Wntregulated MYC mRNA expression (Wnt/ $\beta$-catenin) and Wnt/GSK3regulated MYC protein stability, we generated HPAF-II cell lines stably overexpressing either $M y c$ (MYC OE) or GSK3-resistant $M y c$ (MYC T58A) under the control of the Wnt-independent CMV promoter. The CMV promoter drove 10- to 15-fold higher Myc mRNA expression in orthotopic tumors (Figure 5A). We then compared the effect of PORCN inhibition on the growth of HPAF-II, HPAF-II (MYC OE), and HPAF-II (MYC T58A) orthotopic xenografts.

The MYC OE orthotopic tumors showed a marked increase in MYC protein, but there was no overall increase in tumor growth, and remarkably, they still responded significantly to PORCN inhibition (Figure 5, B and C). Thus, restoration of MYC by overexpression does not rescue tumors from the effects of Wnt inhibition. This could be either because MYC protein is not rate limiting for tumor growth in this setting or because PORCN inhibition was able to 
A

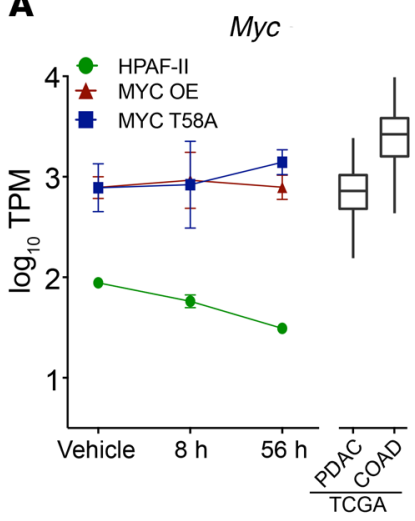

B

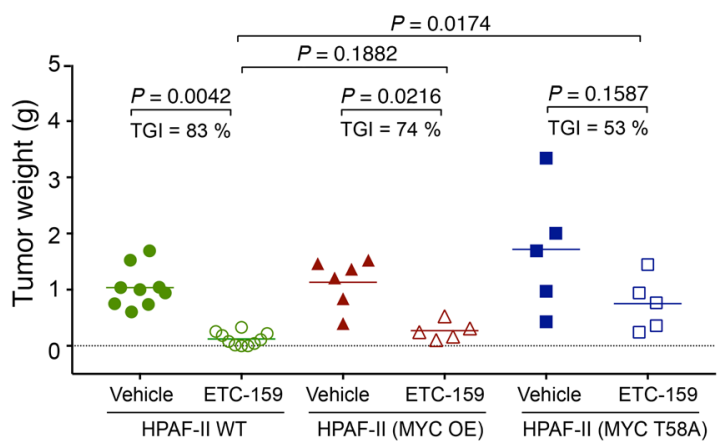

C

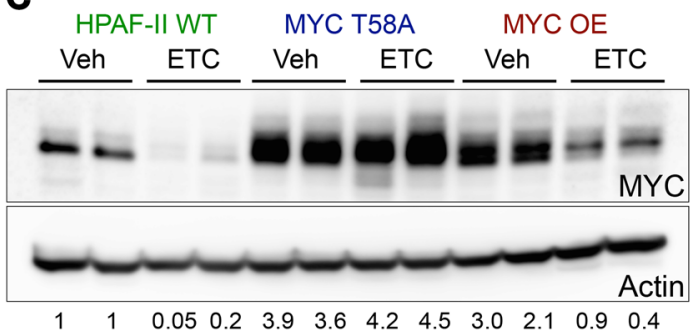

Figure 5. Stabilized MYC partially reverses the effects of Wnt inhibition. (A) Expression of nonendogenous Myc transcripts in MYC OE or MYC T58A tumors does not change with ETC-159 treatment, in contrast to the response of the endogenous MYC transcript in the HPAF-II tumors. Right: range of MYC expression in clinical pancreatic ductal adenocarcinomas (PDAC) and colon adenocarcinomas (COAD) sequenced as part of the TCCA. The Myc expression in MYC OE is comparable to the range observed in clinical samples. (B) MYC T58A cancers are partially responsive to Wnt inhibition. HPAF-II cells, MYC OE, or MYC T58A HPAF-II cells were injected into the pancreas as before. Following the establishment of tumors, mice were treated daily with $30 \mathrm{mg} / \mathrm{kg}$ ETC-159. Tumor weights after 28 days of treatment are shown. $n=5-9$ mice/group. Overall differences were assessed using nonparametric 2-way ANOVA (condition, $P=0.0056$; treatment, $P<2 \times 10^{-16}$ ). Pairwise differences between conditions were assessed using post hoc Mann-Whitney $U$ test ( 2 tailed). TGI, tumor growth inhibition. (C) Ectopically expressed MYC is sensitive to CSK3-mediated degradation. ETC-159 treatment reduces the protein abundance of both endogenous and ectopically expressed MYC in HPAF-II and MYC OE tumors. Mutation of the GSK3 phosphorylation site prevents the decrease in MYC protein abundance in response to Wnt inhibition in the HPAF-II (T58A) tumors. Ratios of MYC levels to $\beta$-actin levels for each lane are indicated.

drive MYC degradation. Consistent with this Wnt/STOP effect, we found that PORCN inhibition caused a decrease in MYC protein abundance despite no change in ectopic MYC mRNA levels (Figure $5, \mathrm{~A}$ and $\mathrm{C}$ ).

We next determined whether blocking the Wnt/STOP effect on MYC protein altered the response to PORCN inhibition. The abundance of MYC T58A did not change upon PORCN inhibition (Figure 5C), and tumors with stabilized MYC grew larger and showed a partial response to PORCN inhibition (Figure 5B). Taken together, these findings indicate that, in addition to regulating MYC transcription, inhibiting Wnt signaling regulates the growth of the tumors by directly regulating MYC protein abundance via a GSK-dependent mechanism. Further, the finding that tumors with stabilized and overexpressed MYC still partially respond to PORCN inhibition shows that Wnts regulate the growth of pancreatic tumors via both Myc-dependent and Myc-independent pathways. This is similar to findings in the murine intestine, in which MYC is essential for the oncogenic effects of APC deletion (36), but alone is insufficient to drive tumorigenesis (37).

Distinguishing between Myc-dependent and Myc-independent regulation of Wnt target genes. To directly identify MYC-independent and MYC-dependent WNT target genes, we performed an additional set of RNA-Seq experiments to examine gene expression changes in orthotopic tumors generated from HPAF-II WT, MYC OE, or MYC T58A cells. We selected as the time points 0,8 , and 56 hours after the start of therapy to allow us to examine early direct targets of both Wnt/ $\beta$-catenin and Wnt/GSK3/MYC signaling. Consistent with the previous experiment, ETC-159 treatment reduced MYC protein by approximately $70 \%$ in 56 hours in HPAF-II WT tumors, while protein levels in MYC T58A tumors showed no decrease (Figure 6A).

We identified 2,131 genes whose transcriptional response to PORCN inhibition in vivo was dependent on MYC status (FDR $<10 \%$, Supplemental Table 5). These genes, whose response to PORCN inhibition was different among WT, MYC OE, or MYC T58A tumors, were classified as MYC-dependent Wnt target genes. Of these genes, 827 (23\%) were found in our set of Wnt-activated genes. For each of the clusters of Wnt-activated genes, we determined the fraction that exhibited MYC-dependent or MYC-independent responses (Figure 6B). The majority of genes that were downregulated most rapidly upon Wnt inhibition (i.e., C9, C10, $\mathrm{C} 20$, and $\mathrm{C} 25 ; \mathrm{TI}_{50}<20$ hours) (Figure 2A) were MYC independent. Selected examples of well-established Wnt-regulated MYC-independent genes, such as AXIN2 and NKD1, are illustrated in Figure 6D, which examines both the relative transcript abundance and the log fold changes. Not surprisingly, the MYC-independent Wnt target genes in C9 were associated with Wnt-signaling pathways and embryonic patterning (Figure 6C).

Only third wave clusters $\mathrm{C} 5$ and $\mathrm{C} 1$, changing with $\mathrm{TI}_{50}$ of 20.6 and 26.5 hours, contained a sizeable fraction ( $>25 \%)$ of MYCdependent genes (Figure 6B) that were enriched in ribosome biogenesis and cell-cycle processes discussed below. We did not observe enrichment for E-boxes in clusters C1 and C5 (Figure 4A). Interestingly, the subsets of genes in these clusters that were MYC dependent were also not specifically enriched for MYC TFBS, suggesting either that they are indirect targets of MYC or that TFBS analysis is not powerful enough to detect a clear enrichment for these MYC motifs.

Regulation of cell cycle by Wnts in Wnt-addicted cancers is Wnt/ GSK3 dependent. Our initial analysis (Figure 2) demonstrated that cell cycle and ribosome biogenesis are 2 key pathways that are transcriptionally regulated by Wnt inhibition, with multiple 
A

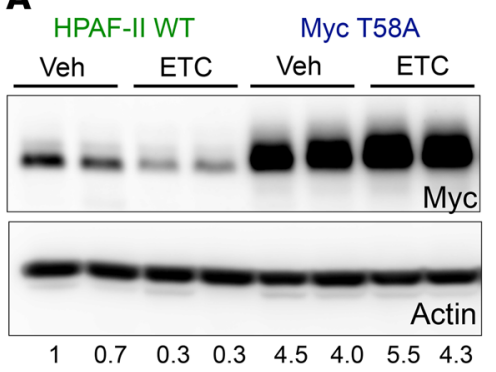

B

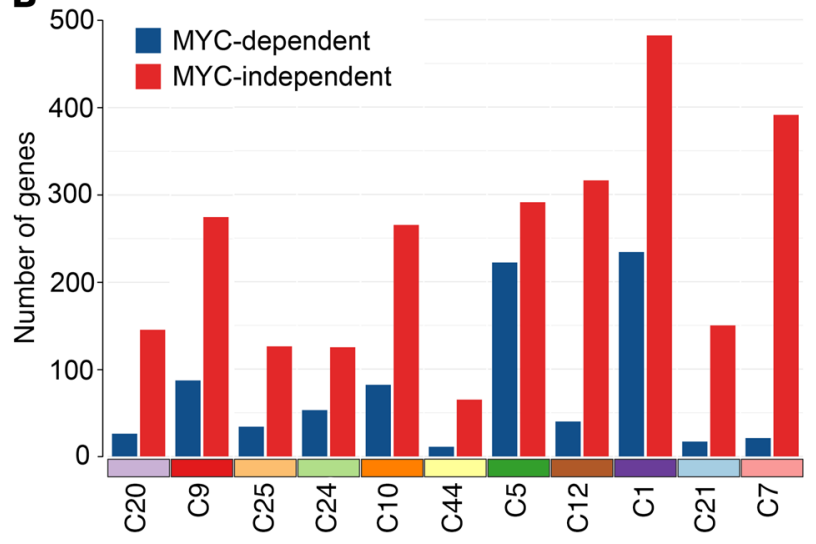

C

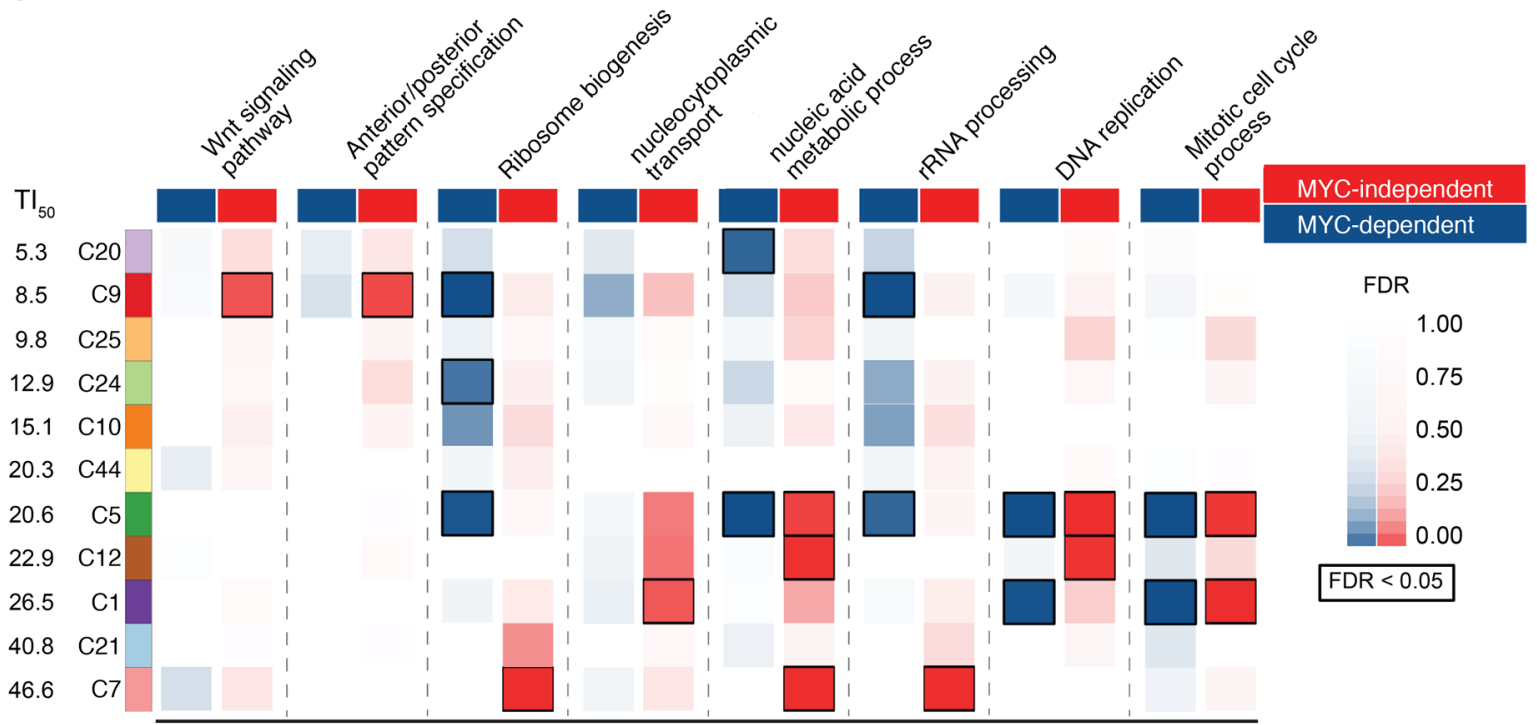

GO biological process

D
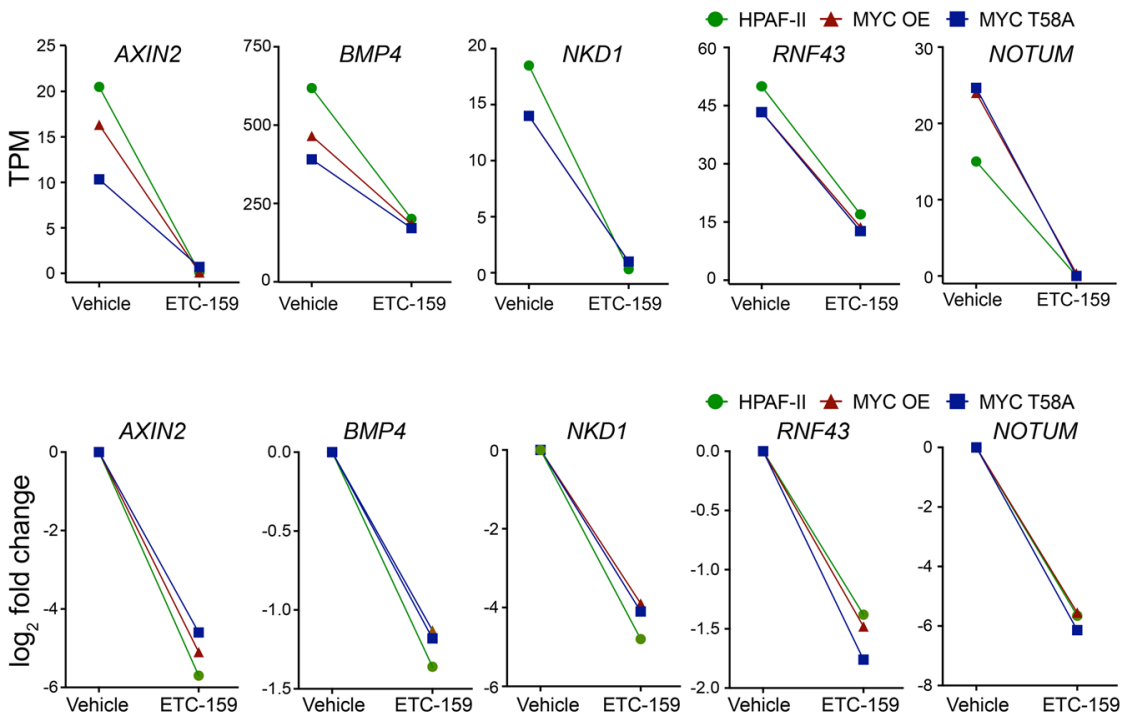

Figure 6. The majority of early responding Wnt-activated genes are MYC independent.

(A) Treatment with ETC-159 for 56 hours reduces the protein abundance of MYC in HPAF-II WT xenografts, but not HPAF-II T58A xenografts. Ratios of MYC levels to $\beta$-actin levels for each lane are indicated. (B) The majority of Wntactivated genes are MYC independent. Whtactivated genes were classified as either MYC dependent or MYC independent based on whether they responded differently to ETC-159 treatment (interaction test, $q$ value $<10 \%$ ) across the 3 xenograft models studied (HPAF-II, MYC OE, and MYC T58A). (C) MYC-dependent and -independent Wnt-activated genes in each time-series cluster regulate distinct biological processes (gene ontology: biological process) (hypergeometric test). Annotated WNT target genes (i.e. Wnt signaling and anterior/posterior patterning) are MYC independent. Ribosome biogenesis and cell-cycle genes are regulated both by MYC-dependent and -independent pathways. (D) Representative examples of early responding MYC-independent Wnt-activated target genes. Expression and $\log _{2}$ fold changes in HPAF-II, MYC $\mathrm{OE}$, and MYC T58A xenograft model systems treated with ETC-159 for 56 hours. 
A
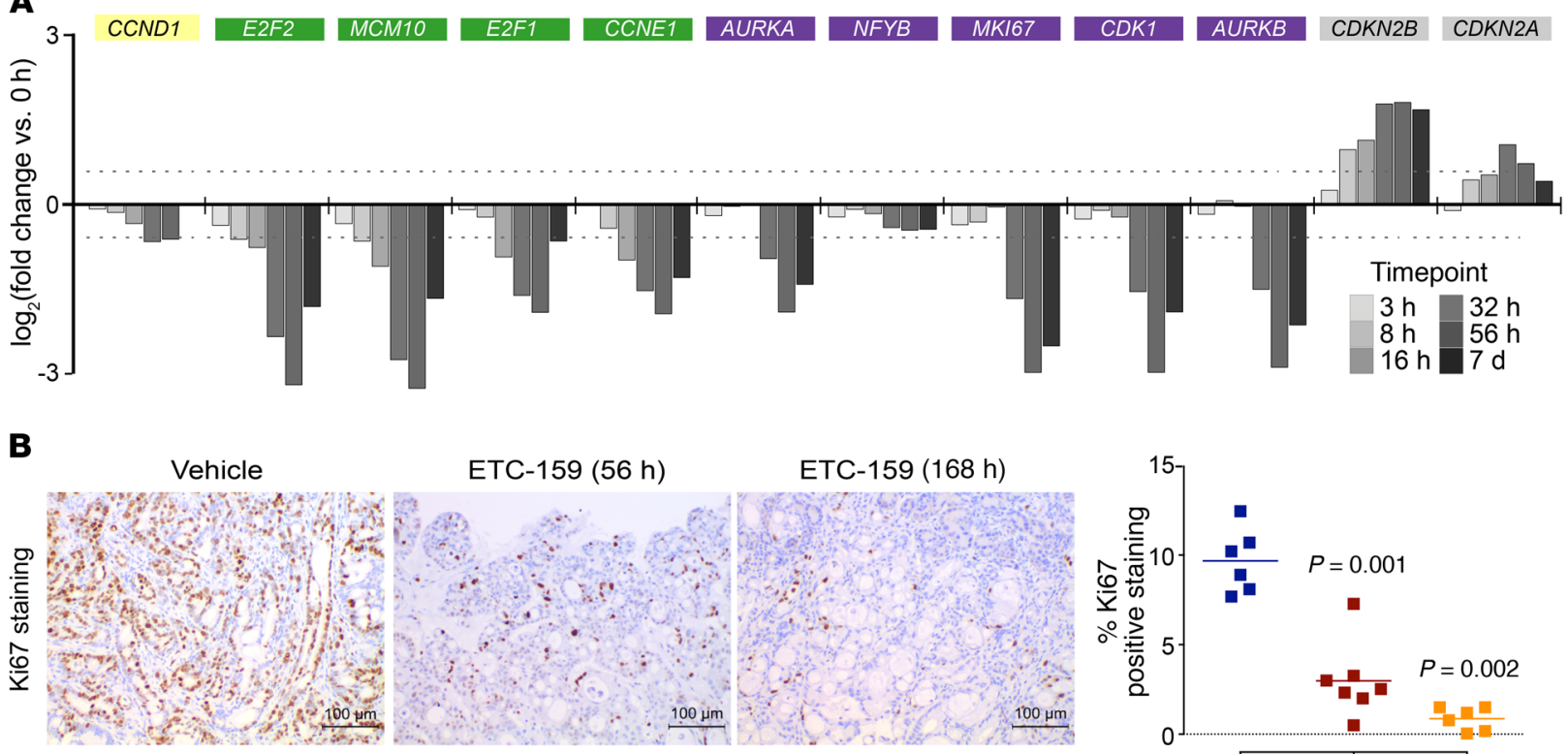

C

HPAF-II

Vehicle ETC-159 (8 h) ETC-159 (56 h)

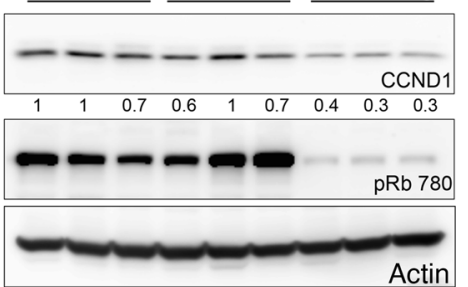

CRC-PDX
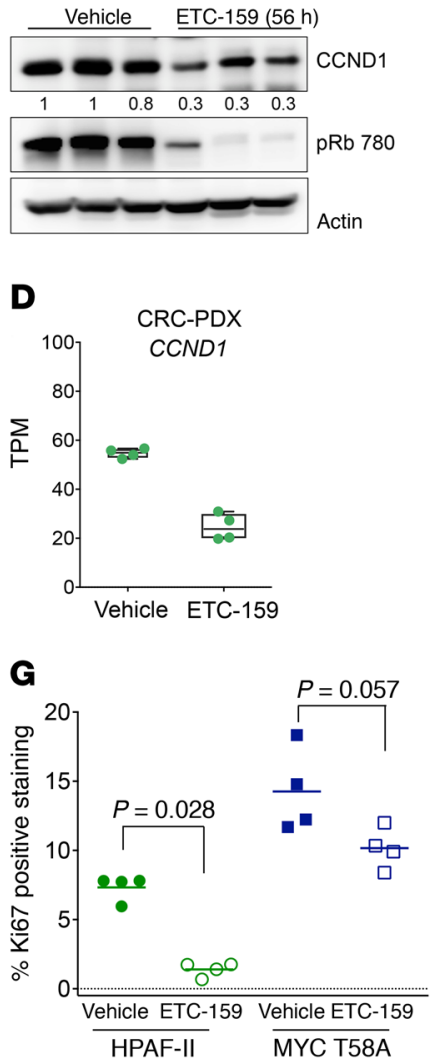

E

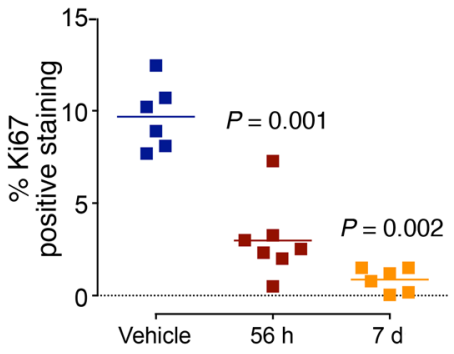

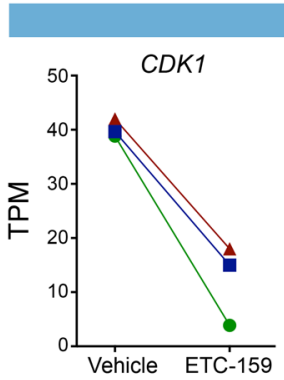
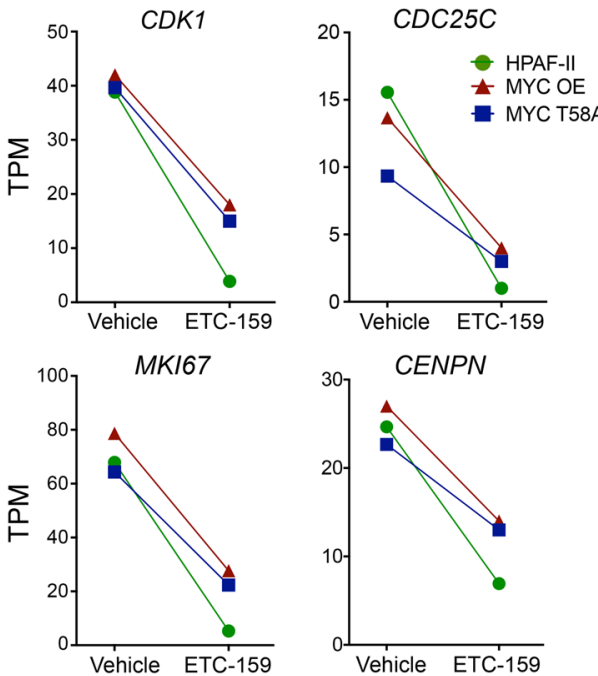

CENPN

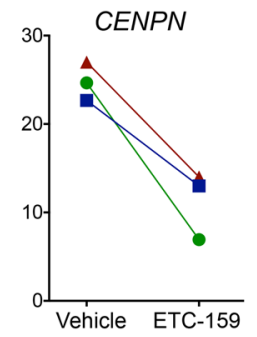

$\mathbf{F}$

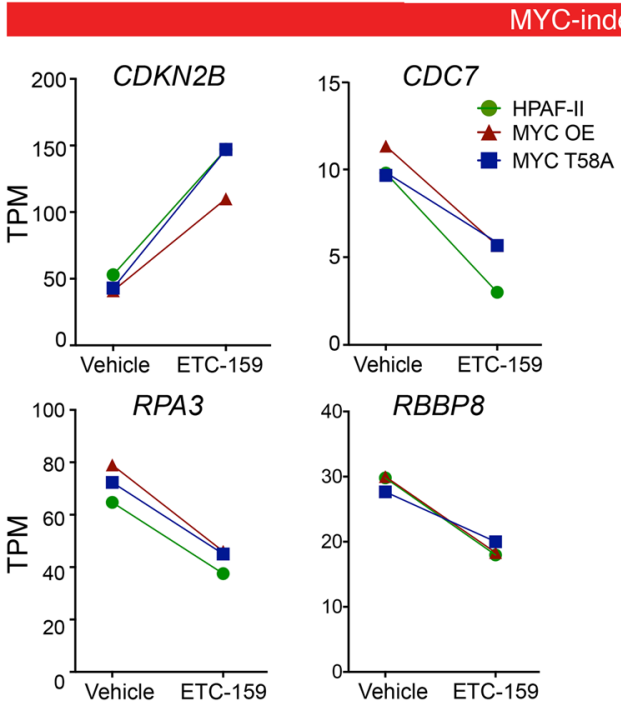

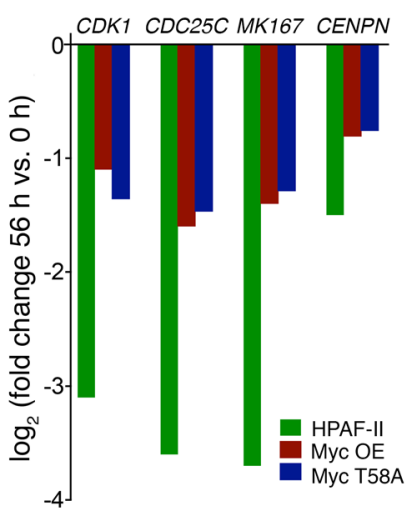


Figure 7. Wnt-regulated cell-cycle changes are only partially influenced by MYC. (A) Robust changes in the expression of representative cell-cycle genes over 7 days of PORCN inhibition in HPAF-II orthotopic xenografts. The dotted line is at 1.5 -fold $\left(\log _{2}, 0.58\right)$. (B) Significant decrease in proliferating cells over time. Ki67-positive cells (left) were quantitated (right) on an entire section that was scanned and analyzed using NIS-Elements software. Two-tailed Mann-Whitney $U$ test was used to calculate significant differences. (C) CCND1 protein changes more robustly compared with mRNA. Blots from Figure 4C were reprobed as indicated. CCND1 mRNA decreases 33\% (see $\mathbf{A}$ and $\mathbf{D}$ ), while CCND1 protein decreases approximately $65 \%$ after 56 hours treatment in both HPAF-II and CRC PDX models. Each lane is from an independent tumor. Ratios of CCND1 levels to $\beta$-actin levels for each lane are indicated. ETC-159 treatment reduces levels of pRb (S780) in both HPAF-II tumors and CRC PDX. Note the actin blots are the same as in Figure 4C. (D) Changes in the expression of CCND1 after 56 hours in CRC PDX model. (E) Representative cell-cycle genes whose expression is influenced by MYC overexpression. Relative expression is plotted (TPM) (left panel) or as $\log _{2}$ fold change (right panel). (F) Representative cell-cycle genes whose expression is independent of MYC overexpression. Relative expression is plotted as TPM (left panel) or as $\log _{2}$ fold change (right panel). (C) MYC stabilization only partially rescues proliferation upon PORCN inhibition. Ki-67 staining quantitated as in B. Two-tailed Mann-Whitney $U$ test was used to calculate significant differences.

genes regulating cell cycle changing in a time-dependent manner, including CDK1, E2F2, E2F1, CDKN2B, and CDKN2A (Figure 7A). Consistent with this robust regulation of cell-cycle genes, Ki67-positive cells were significantly reduced in the tumors as early as 56 hours after starting ETC-159 and were further reduced at 7 days of treatment (Figure 7B).

Genes associated with mitotic cell-cycle processes and DNA replication were enriched in the third wave of clusters (C1, C5, $\mathrm{C} 12 ; \mathrm{TI}_{50}, 20.6-26.5$ hours) (Figure 2B). These clusters were enriched for binding sites for the E2F and NFY families of transcription factors (Figure 4A) that cooperatively regulate cell-cycle genes $(32,38,39)$. Interestingly, $E 2 F 1$ and $E 2 F 2$ gene expression decreased at the same rate as that of the other cell-cycle genes, suggesting that the early decrease in the expression of cell-cyclerelated genes was not due to changes in these E2F mRNAs (Figure 7A). E2F activity is also regulated by cyclin-dependent kinase (CDK) signaling through $\mathrm{p} 105 / \mathrm{Rb}$. Indeed, the expression of the CDK inhibitors increased as early as 8 hours after PORCN inhibition in orthotopic tumors, and this was associated with a subsequent decrease in $\mathrm{Rb}$ phosphorylation (Figures 7, $\mathrm{A}$ and $\mathrm{C}$ ). Thus, $\mathrm{CDK}$ inhibition and decreased $\mathrm{Rb}$ phosphorylation are likely to be major mechanisms driving the decrease in the transcription of E2F target cell-cycle genes.

Notably, the abundance of cell-cycle regulators, such as cyclin D1 and E1, among others, is also regulated by Wnt/STOP signaling (8). Similarly to MYC, after 56 hours of ETC-159 treatment, the protein abundance of CCND1 was reduced by approximately 3-fold (Figure 7C), while CCND1 transcript levels were only reduced by approximately 1.5 -fold in both HPAF-II xenografts and CRC PDX (Figure 7, A and D). Thus, our in vivo data in Wnt-driven cancers support the data from in vitro studies (8) showing that Wnt signaling regulates CCND1 and MYC by both transcriptional and posttranscriptional mechanisms.

We further examined the role of MYC in the regulation of cell-cycle genes. Notably, MYC overexpression had no effect on baseline expression of the cell-cycle genes and Wnt inhibition reduced their expression, albeit to differing extents even in the presence of stabilized MYC (Figure 7, E and F). Stabilized MYC blunted the effect of PORCN inhibition on the expression of a subset of the cell-cycle genes, e.g., CDK1 and MKI67 (Figure $7 \mathrm{E}$ ). However, a number of other cell-cycle genes (e.g. CDKN2B, $C D C 7, R B B P 8$, and RPA3) were MYC independent and responded to Wnt inhibition even in MYC-stabilized tumors (Figure $7 F$ ). Consistent with the observed transcriptional response, there was a partial reduction of Ki67 staining in ETC-159-treated MYCstabilized tumors (Figure 7G). Taken together, these findings indicate that Wnt regulates the cell cycle in cancers via multiple pathways, both depending on MYC and independently of it and through both transcriptional and Wnt/STOP mechanisms.

Wnt signaling regulates ribosome biogenesis. The enrichment for rRNA processing and ribosome biogenesis in the Wnt-activated gene clusters (Figure 2B) suggested that PORCN inhibition would lead to a reduction in ribosome formation and protein synthesis. Indeed, nearly all genes encoding ribosomal protein subunits (RPSs and RPLs) were downregulated (Figure 8A), with 94\% of differentially regulated RPSS and RPLs being present in lateresponding clusters, $\mathrm{C} 7$ ( $\mathrm{TI}_{50}, 46.6$ hours) or $\mathrm{C} 1$ ( $\mathrm{TI}_{50}, 26.5$ hours). Although the expression of RPS and RPL genes was reduced by only approximately $30 \%-40 \%$, the changes were largely coherent, albeit with some outliers of unknown significance. The changes were more apparent following 32 hours of treatment, suggesting that these genes are indirectly regulated by Wnt signaling. We next determined whether this was reflected in the abundance of ribosomal subunit proteins. In a parallel mass spectrometry experiment that only detected high-abundance proteins (see Methods), we confirmed that the RPS and RPL proteins were also coherently downregulated at 56 hours (Figure 8B and Supplemental Table 6). Given the high abundance of ribosomal proteins, this suggests a dramatic shift in ribosome biogenesis.

Ribosomal biosynthesis requires multiple processes, including nucleocytoplasmic transport and rRNA expression and processing (30). We found that genes required for nucleocytoplasmic export, including exportins and nucleoporins, were similarly coherently downregulated, implicating the regulation of ribosome assembly by Wnt signaling (Figure 8C). Multiple components of the machinery required for $\mathrm{rRNA}$ transcription, including several subunits of RNA polymerases POLR1 and POLR3 (Figure $8 \mathrm{D}$ ) and rRNA processing factors (e.g., NPM1, DKC1), were also downregulated (Figure $8 \mathrm{E})$. Finally, consistent with the changes in gene and protein expression, the size of nucleolar organizer regions was reduced by ETC-159 treatment (Figure 9A). Taken together, these data indicate that ribosome biogenesis is globally regulated by Wnt signaling. A global decrease in protein synthesis coupled with a halt in the cell cycle likely explains how PORCN inhibition blocks tumor progression in Wnt-addicted cancers (40).

We asked whether Wnt regulation of ribosome biogenesis was explained by its effect on MYC, a recognized regulator of ribosome biogenesis (30). In contrast to what occurs with cell-cycle genes, the baseline expression of ribosome subunit and biogenesis genes was increased by stabilized MYC (Figure 9, B and C). However, a number of these genes remained sensitive to PORCN inhibition and decreased after 56 hours of ETC- 
A

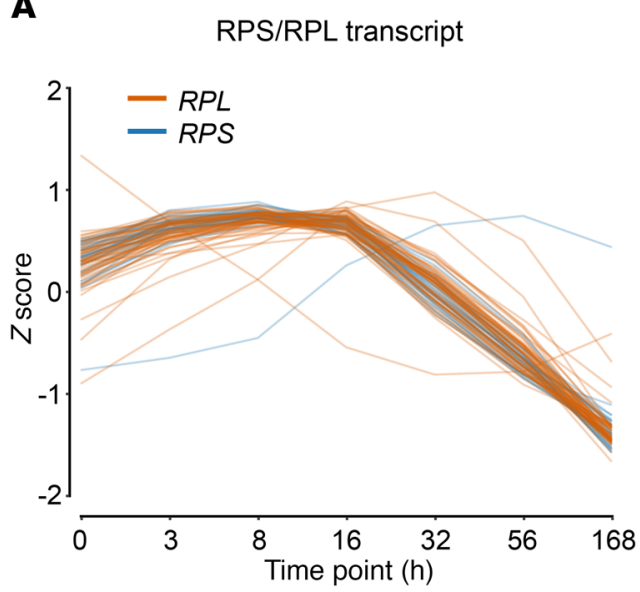

B

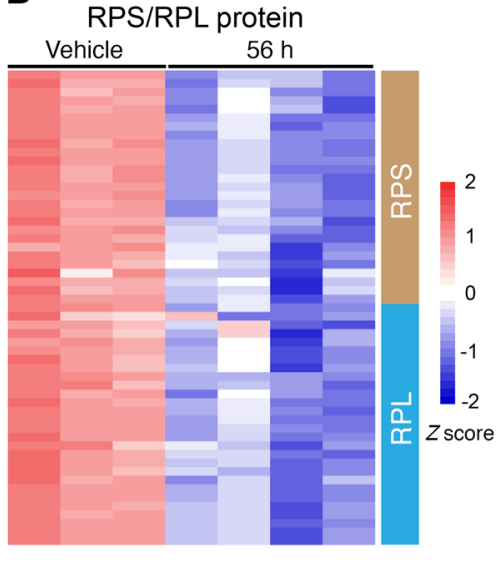

D
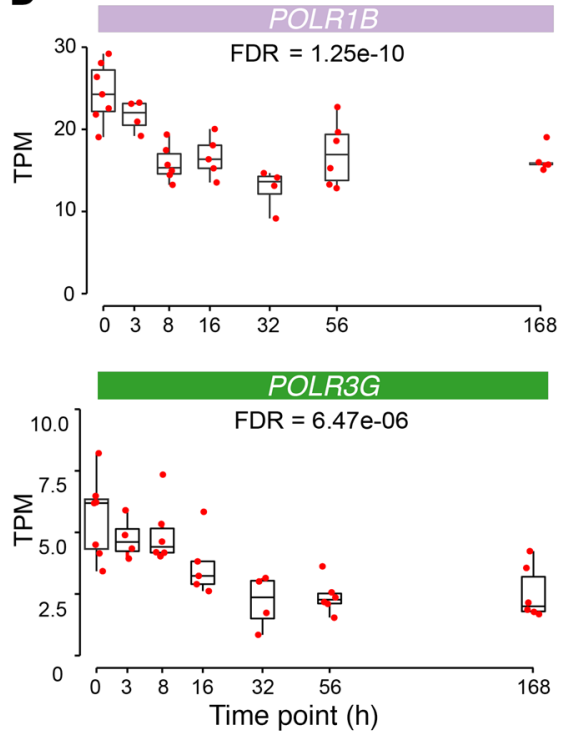

E
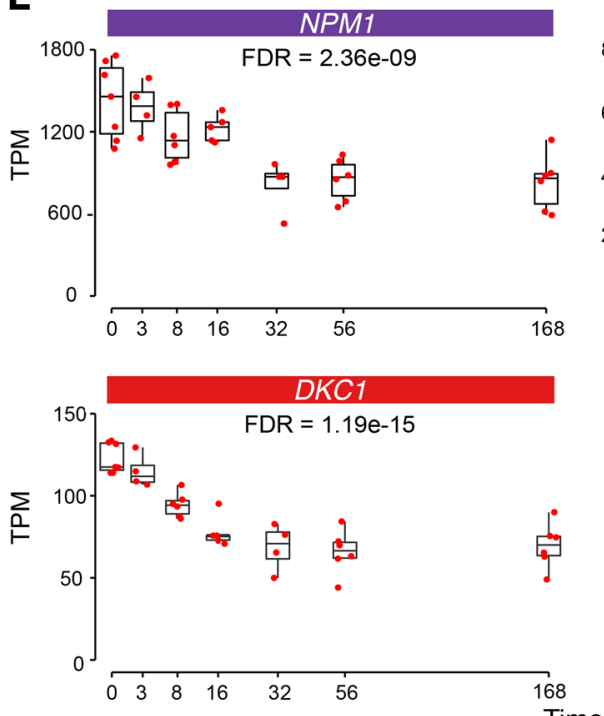

C

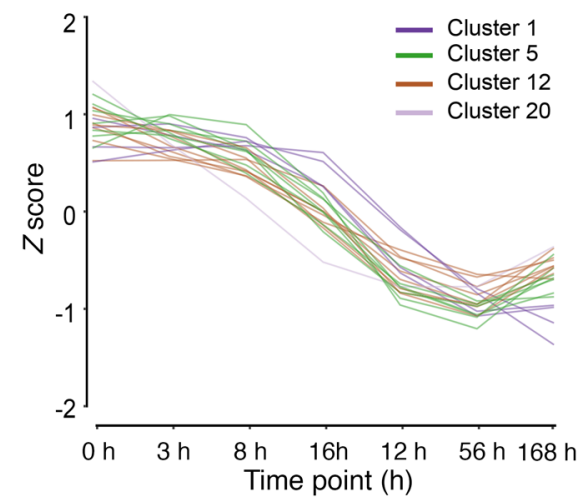

Nucleoporins
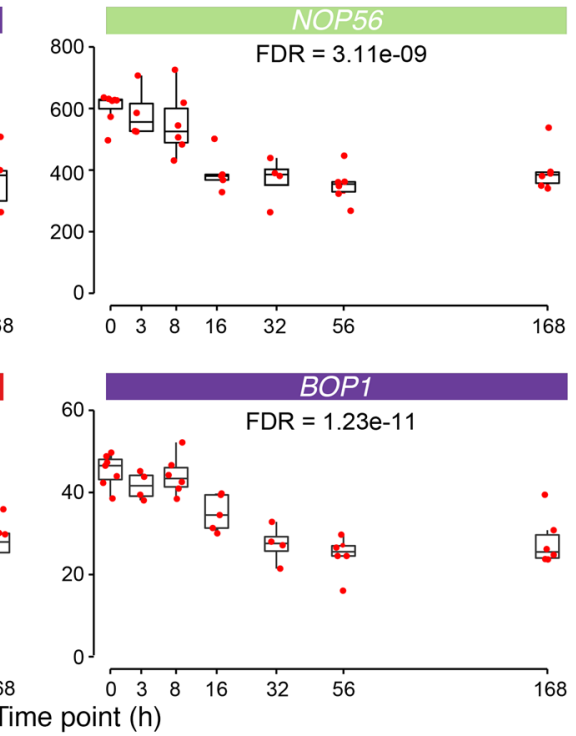

Figure 8. PORCN inhibition leads to a reduction in expression of key ribosome biogenesis genes. (A) Coherent changes in the expression of genes encoding ribosomal proteins (RPLS/RPSs) over time following PORCN inhibition. (B) Coherent changes in the abundance of ribosomal proteins over time as assessed by mass spectrometry following PORCN inhibition. (C) Coherent changes in the expression of nucleoporin genes over time following PORCN inhibition. (D) Gene expression of RNA polymerases subunits, including POLR1B and POLR3G, that transcribe ribosomal RNA are Wnt regulated. (E) Key regulators of ribosome biogenesis NPM1, BOP1, NUP58, and DKC1 change over time following PORCN inhibition.

159 treatment even in cells with MYC T58A (Figure 9C). These Wnt-regulated, Myc-independent ribosome genes include virtually all of the RPLs and RPSs (C1 and C7) (Figure 9B and Supplemental Figure 5). Another subset of genes involved in rRNA synthesis and processing (e.g., NPM1, DKC1, POLR1B) were MYC-dependent WNT target genes. These genes were both highly MYC responsive at baseline and, consistent with their dependency from Wnt/STOP regulation of MYC protein abundance, did not respond to Wnt inhibition when MYC T58A was present (Figure 9C). These MYC-dependent genes are enriched for E-boxes in their promoters.

Our analysis thus establishes a key role of Wnt signaling in ribosome biogenesis via 2 routes. One route, via MYC, is regulated both through Wnt-driven MYC expression and via the Wnt/STOP pathway. The other route is MYC independent and is a downstream effect of WNT signaling on the transcription of ribosomal genes.

\section{Discussion}

The development of targeted drugs that rapidly and robustly inhibit PORCN provides a unique opportunity to examine in real time the consequences of Wnt withdrawal in Wnt-addicted human cancers. This time-based analysis of Wnt signaling and its interaction with MYC provides a comprehensive assessment of the role played by Wnt ligands in driving Wnt-addicted cancer. Importantly, the high concordance of the transcriptional changes in Wnt-addicted RSPO3-mutant colorectal and RNF43-mutant pancreatic cancers reveals core shared pathways regulated by Wnt signaling in cancer. Previous studies examining the targets of Wnt signaling in cancer have focused on models that are driven by loss-of-function mutations in APC. Here, the use of Wnt ligand-driven cancer mouse models casts a broader net, identifying an unexpectedly large number of genes whose expression depends on the continued presence of the Wnt ligand, many of which are independent of $\beta$-catenin. 
A

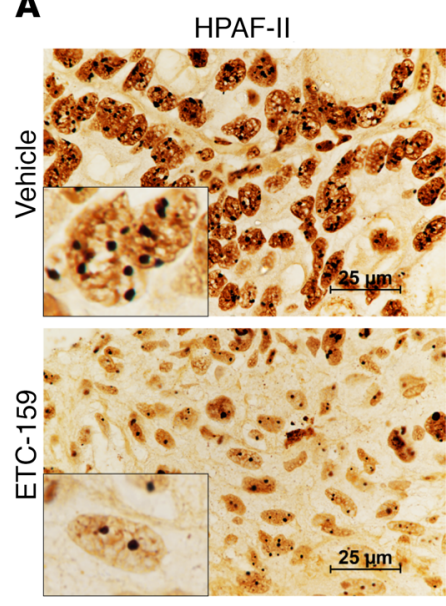

C
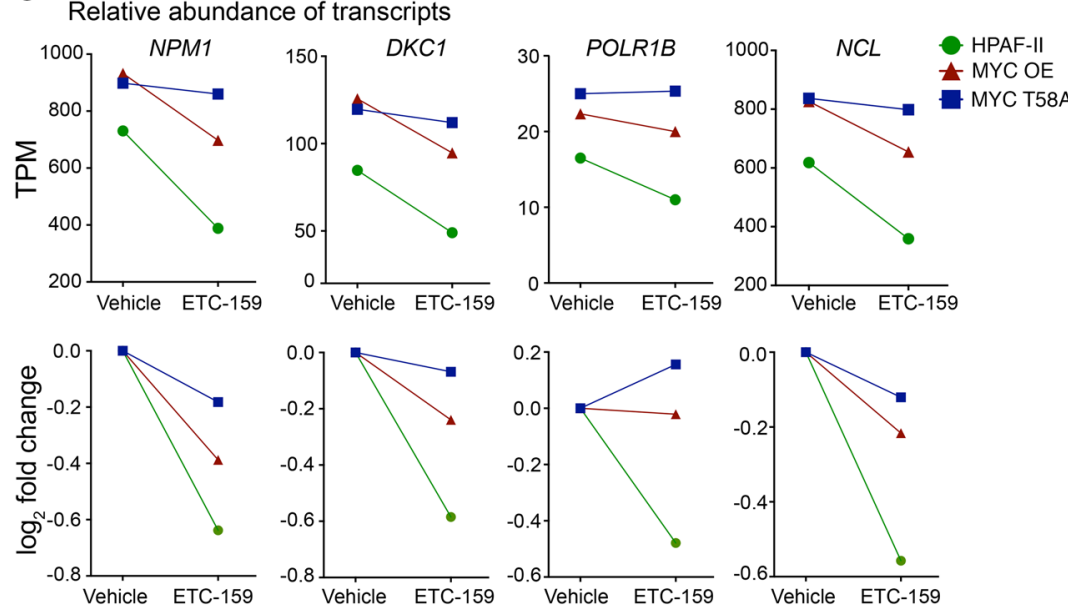

MYC T58A

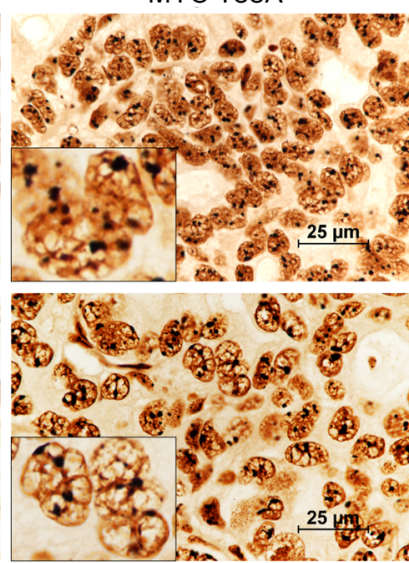

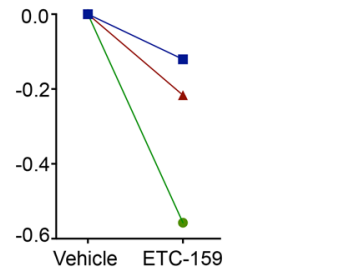

MYC OE

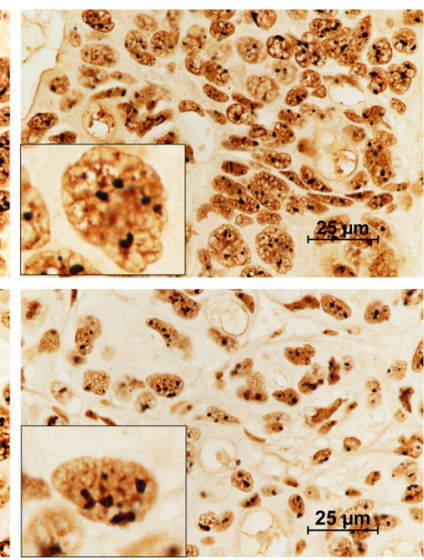

B

RPS/RPL transcripts

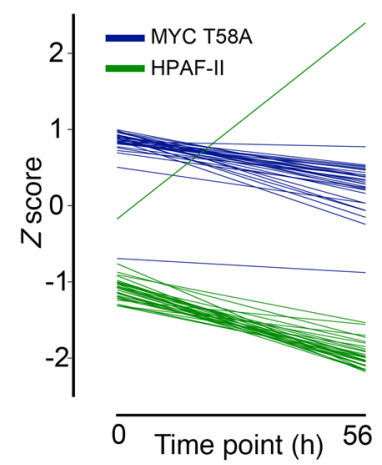

Figure 9. Wnt and MYC coregulate ribosome biogenesis. (A) Wnt inhibition reduces nucleoli, but this is rescued by expression of stabilized MYC. Changes in nucleolar size and abundance in tumor sections was assessed by silver staining. (B) Expression of ribosomal subunit genes (RPLs/RPSs) is enhanced by stabilized MYC T58A, but remains sensitive to PORCN inhibition. The outlier in HPAF-II tumors is RPS27L, as in Figure 8A. (C) Representative Wnt-activated ribosome biogenesis genes that are MYC dependent. Changes in selected genes in MYC $\mathrm{OE}$ and T58A tumors are shown. Relative expression is plotted as TPM (top panels) or as $\log _{2}$ fold change (bottom panels).

The genes whose expression changes most rapidly after PORCN inhibition, the early wave clusters, were predictably enriched for well-established $\beta$-catenin target genes (41). However, our analysis revealed a large number of coregulated genes that were not known $\beta$-catenin targets. DNA sequence-based analysis of enrichment for TCF/LEF-binding sites was not a useful approach to discriminating between whether these early changing genes could be additional $\beta$-catenin targets or could be regulated by multiple noncanonical pathways. Indeed, while many individual studies found TCF/LEF sites in the promoters of selected genes, our findings support the results from genome-wide analyses showing that functional TCF/ LEF sites are often present at large distances from transcriptional start sites (3). Additionally, recent studies have established that even $\beta$-catenin promoter binding is not sufficient for identifying $\beta$-catenin transcriptionally regulated genes (5).

Interestingly, our analysis revealed that E-box TFBSs are enriched in the early changing genes, followed at later time points by enrichment for E2F-binding sites. Finally, the fourth wave of genes was enriched for a broader set of TFBS that are likely to be regulated as secondary, downstream events. The enrichment for E-boxes strongly suggested a role for MYC. MYC is a potent oncogene, and its activation is a hallmark of cancer initiation and maintenance $(42,43)$. MYC is required for tumorigenesis following $\beta$-catenin activation by APC loss in the gut, but not in the liver
$(36,44)$. Hence, it was an open question whether MYC would be important downstream of RNF43 mutations in pancreatic cancers, where many additional pathways are activated by the Wnt addiction $(36,45)$. Using a model of Wnt-addicted human cancer with stabilized MYC, we were able to disentangle the interaction of Wnt and MYC and stratify the roles of Wnts and MYC in regulating cell cycle and ribosome biogenesis. One notable difference was that stabilization of MYC did not enhance the expression of cell-cycle genes. However, stabilized MYC could partially overcome the effect of Wnt inhibition on expression of a subset of cell-cycle genes (46), whereas $M y c$ overexpression and stabilization more profoundly affected genes regulating various processes associated with ribosome biogenesis. Here too, the response to Wnt inhibition was variable, as a large subset of genes, including ribosomal proteins, responded to PORCN inhibitors with similar fold changes, while others were "immune" to Wnt inhibition in the presence of stabilized MYC. This suggests a complex interaction of MYC and Wnt-regulated pathways driving these processes.

Ribosomes are overexpressed in cancer and have become targets for anticancer therapies, for instance, by triggering nucleolar stress (40, 47-49). While MYC is known to regulate ribosome biogenesis (30), the role of Wnts has been less clear $(50,51)$. Here, we show for what we believe is the first time that Wnt signaling globally affects multiple steps in ribosome biogenesis both directly 
and by regulating the transcription and protein abundance of Myc via the Wnt/STOP pathway and that this is shared in both Wnt-addicted pancreatic and colorectal cancers.

Comparing the effect of PORCN inhibition across different models confirmed the value of studying Wnt signaling in an orthotopic microenvironment or in the present of native stroma (CRC PDX). The experimental value of the orthotopic model using a cell line is that it is more amenable to genetic manipulation, such as the introduction of stabilized MYC, allowing a more detailed analysis of the role of downstream drivers.

The stabilization of MYC via the Wnt/GSK3 signaling axis highlights how this mechanism can target MYC and other cell-cycle proteins in cancer, affecting aberrant cell growth (52). The Wnt/STOP pathway is likely to have multiple additional targets $(9,10)$ that may also play a role in these pancreatic and colorectal cancers. Future studies with high-resolution mass spectrometry at early time points after Wnt inhibition may facilitate their identification. The data provided in this study can facilitate biomarker discovery for patients suffering from Wnt-addicted cancers and provide a significant resource for the Wnt and cancer community.

\section{Methods}

Tumor growth and mouse treatment. Mouse xenograft models from HPAF-II cells were established by orthotopic injection of HPAF-II cells in NOD SCID gamma mice as described in the Supplemental Methods. Mice were purchased from InVivos or Jackson Laboratories. Animals were housed in standard cages and were allowed ad libitum access to food and water.

Western blot analysis. Tumors were homogenized in 4\% SDS buffer, and proteins were resolved on 10\% SDS-polyacrylamide gel. Western blots were performed according to standard methods.

Immunohistochemistry and AgNOR staining. Formalin-fixed and paraffin-embedded tissue sections were then stained with H\&E, Ki67, or nucleolar organizer regions using standard protocol. Images were acquired using a Nikon E microscope.

RNA isolation and data analysis. Tumors were homogenized in RLT buffer, and total RNA was isolated using the RNeasy Kit (QIAGEN) according to the manufacturer's protocol. The RNA-Seq libraries were prepared using the Illumina TruSeq Stranded Total RNA protocol with subsequent PolyA enrichment. Details for quality control and data processing for RNA-Seq, TFBS analysis, time-series clustering, and ChIPSeq analysis are provided in the Supplemental Methods.
Proteomics. Tumors were homogenized on dry ice and solubilized with $8 \mathrm{M}$ urea and $20 \mathrm{mM}$ HEPES. Trypsin-digested peptides in $0.1 \%$ trifluoroacetic acid (TFA) were separated using an Ultimate 3000 RSLC Nano Liquid Chromatography System coupled to a Q-Exactive Mass Spectrometer (Thermo Fisher Scientific). Following datadependent acquisition, raw data files were loaded and analyzed using Progenesis QI (Nonlinear Dynamics).

Statistics. Statistical analysis was performed using R and GraphPad. A $P$ value of less than 0.05 was considered significant. For genome-wide analysis, an FDR of less than $10 \%$ was considered significant, and for enrichment analyses, an FDR of less than $5 \%$ was considered significant.

Study approval. The SingHealth Institutional Animal Care and Use Committee approved all animal studies, which complied with applicable regulations.

Data availability. The RNA-Seq data were deposited in the NCBI's Gene Expression Omnibus database (GEO GSE118041, GSE118231, GSE118190, and GSE118179).

\section{Author contributions}

$\mathrm{BM}, \mathrm{NH}, \mathrm{EP}$, and DMV designed the study. BM and GN performed the animal studies and biochemical analysis. NH designed and performed the bioinformatics analysis. AM and PF performed mass spectrometry. EP and DMV supervised the study. BM, $\mathrm{NH}$, $\mathrm{EP}$, and DMV wrote the manuscript.

\section{Acknowledgments}

We acknowledge the assistance of members of the Virshup lab and members of the Experimental Therapeutics Centre. We acknowledge Ralph Bunte for his expert advice with histological analysis and the assistance of the vivarium staff, including Hock Lee. This research is supported in part by the National Research Foundation Singapore and administered by the Singapore Ministry of Health's National Medical Research Council under the STAR Award Program (to DMV).EP acknowledges the support of the MRC London Institute of Medical Sciences, Imperial College, London. BM acknowledges the support of the Singapore Ministry of Health's National Medical Research Council Open Fund-Independent Research Grant.

Address correspondence to: David M. Virshup or Enrico Petretto, Duke-NUS Medical School, 8 College Road, Singapore, Republic of Singapore 169857. Phone: 65.6516.7881; Email: david.virshup@duke-nus.edu.sg (D.M. Virshup). Phone: 65.66015114; Email: enrico.petretto@duke-nus.edu.sg (E.Petretto).
1. Nusse R, Clevers H. Wnt/ $\beta$-catenin signaling, disease, and emerging therapeutic modalities. Cell. 2017;169(6):985-999.

2. Acebron SP, Niehrs C. $\beta$-Catenin-independent roles of Wnt/LRP6 signaling. Trends Cell Biol. 2016;26(12):956-967.

3. Ramakrishnan AB, Cadigan KM. Wnt target genes and where to find them. F100ORes. 2017;6:746.

4. Yu J, Virshup DM. Updating the Wnt pathways. Biosci Rep. 2014;34(5): e00142.

5. Nakamura Y, de Paiva Alves E, Veenstra GJ, Hoppler S. Tissue- and stage-specific Wnt target gene expression is controlled subsequent to $\beta$-catenin recruitment to cis-regulatory modules. Develop- ment. 2016;143(11):1914-1925.

6. Ju X, Ishikawa TO, Naka K, Ito K, Ito Y, Oshima M. Context-dependent activation of Wnt signaling by tumor suppressor RUNX3 in gastric cancer cells. Cancer Sci. 2014;105(4):418-424.

7. Chien AJ, Conrad WH, Moon RT. A Wnt survival guide: from flies to human disease. J Invest Dermatol. 2009;129(7):1614-1627.

8. Acebron SP, Karaulanov E, Berger BS, Huang YL, Niehrs C. Mitotic wnt signaling promotes protein stabilization and regulates cell size. Mol Cell. 2014;54(4):663-674.

9. Taelman VF, et al. Wnt signaling requires sequestration of glycogen synthase kinase 3 inside multive- sicular endosomes. Cell. 2010;143(7):1136-1148.

10. Koch S, Acebron SP, Herbst J, Hatiboglu G, Niehrs C. Post-transcriptional Wnt signaling governs epididymal sperm maturation. Cell. 2015;163(5):1225-1236.

11. Zhang X, et al. Mechanistic insight into Myc stabilization in breast cancer involving aberrant Axin1 expression. Proc Natl Acad Sci U S A. 2012;109(8):2790-2795.

12. Nusse R, Varmus H. Three decades of Wnts: a personal perspective on how a scientific field developed. EMBO J. 2012;31(12):2670-2684.

13. Jiang $X$, et al. Inactivating mutations of RNF 43 confer Wnt dependency in pancreatic ductal 
adenocarcinoma. Proc Natl Acad Sci U S A. 2013;110(31):12649-12654.

14. Ong CK, et al. Exome sequencing of liver fluke-associated cholangiocarcinoma. Nat Genet. 2012;44(6):690-693.

15. Seshagiri S, et al. Recurrent R-spondin fusions in colon cancer. Nature. 2012;488(7413):660-664.

16. Cancer Genome Atlas Research Network. Integrated genomic characterization of pancreatic ductal adenocarcinoma. Cancer Cell. 2017;32(2):185-203.e13.

17. Madan B, Virshup DM. Targeting Wnts at the source--new mechanisms, new biomarkers, new drugs. Mol Cancer Ther. 2015;14(5):1087-1094.

18. Madan B, et al. Wnt addiction of genetically defined cancers reversed by PORCN inhibition. Oncogene. 2016;35(17):2197-2207.

19. Rios-Esteves J, Resh MD. Stearoyl CoA desaturase is required to produce active, lipid-modified Wnt proteins. Cell Rep. 2013;4(6):1072-1081.

20. Proffitt KD, et al. Pharmacological inhibition of the Wnt acyltransferase PORCN prevents growth of WNT-driven mammary cancer. Cancer Res. 2013;73(2):502-507.

21. Janda CY, Waghray D, Levin AM, Thomas C, Garcia KC. Structural basis of Wnt recognition by Frizzled. Science. 2012;337(6090):59-64.

22. Takada R, et al. Monounsaturated fatty acid modification of Wnt protein: its role in Wnt secretion. Dev Cell. 2006;11(6):791-801.

23. Coombs GS, et al. WLS-dependent secretion of WNT3A requires Ser209 acylation and vacuolar acidification. J Cell Sci. 2010;123 (Pt 19):3357-3367.

24. Liu J, et al. Targeting Wnt-driven cancer through the inhibition of porcupine by LGK974. Proc Natl Acad Sci U S A. 2013;110(50):20224-20229.

25. Chen B, et al. Small molecule-mediated disruption of Wnt-dependent signaling in tissue regeneration and cancer. Nat Chem Biol. 2009;5(2):100-107.

26. Killion JJ, Radinsky R, Fidler IJ. Orthotopic models are necessary to predict therapy of transplantable tumors in mice. Cancer Metastasis Rev. 1998;17(3):279-284.
27. Byrne AT, et al. Interrogating open issues in cancer precision medicine with patient-derived xenografts. Nat Rev Cancer. 2017;17(4):254-268.

28. Hensman J, Rattray M, Lawrence ND. Fast nonparametric clustering of structured timeseries. IEEE Trans Pattern Anal Mach Intell. 2015;37(2):383-393.

29. Giráldez AJ, Copley RR, Cohen SM. HSPG modification by the secreted enzyme Notum shapes the Wingless morphogen gradient. Dev Cell. 2002;2(5):667-676.

30. van Riggelen J, Yetil A, Felsher DW. MYC as a regulator of ribosome biogenesis and protein synthesis. Nat Rev Cancer. 2010;10(4):301-309.

31. Stevens ML, et al. Genomic integration of Wnt/ $\beta$-catenin and BMP/Smad1 signaling coordinates foregut and hindgut transcriptional programs. Development. 2017;144(7):1283-1295.

32. Dolfini $D$, Mantovani R. Targeting the Y/CCAAT box in cancer: YB-1 (YBX1) or NF-Y? Cell Death Differ. 2013;20(5):676-685.

33. Myant K, Sansom OJ. Wnt/Myc interactions in intestinal cancer: partners in crime. Exp Cell Res. 2011;317(19):2725-2731.

34. Arnold HK, et al. The Axin1 scaffold protein promotes formation of a degradation complex for c-Myc. EMBO J. 2009;28(5):500-512.

35. Sears R, Nuckolls F, Haura E, Taya Y, Tamai K, Nevins JR. Multiple Ras-dependent phosphorylation pathways regulate Myc protein stability. Genes Dev. 2000;14(19):2501-2514.

36. Sansom OJ, et al. Myc deletion rescues Apc deficiency in the small intestine. Nature. 2007;446(7136):676-679.

37. Finch AJ, Soucek L, Junttila MR, Swigart LB, Evan GI. Acute overexpression of Myc in intestinal epithelium recapitulates some but not all the changes elicited by Wnt/beta-catenin pathway activation. Mol Cell Biol. 2009;29(19):5306-5315.

38. Ly LL, Yoshida H, Yamaguchi M. Nuclear transcription factor $\mathrm{Y}$ and its roles in cellular processes related to human disease. Am J Cancer Res. 2013;3(4):339-346.

39. van den Heuvel S, Dyson NJ. Conserved functions of the pRB and E2F families. Nat Rev Mol
Cell Biol. 2008;9(9):713-724.

40. Ruggero D, Pandolfi PP. Does the ribosome translate cancer? Nat Rev Cancer. 2003;3(3):179-192.

41. Moon RT, Gough NR. Beyond canonical: the Wnt and $\beta$-catenin story. Sci Signal. 2016;9(422):eg5.

42. Dang CV. Enigmatic MYC conducts an unfolding systems biology symphony. Genes Cancer. 2010;1(6):526-531.

43. Gabay M, Li Y, Felsher DW. MYC activation is a hallmark of cancer initiation and maintenance. Cold Spring Harb Perspect Med. 2014;4(6):a014241.

44. Reed KR, et al. B-catenin deficiency, but not Myc deletion, suppresses the immediate phenotypes of APC loss in the liver. Proc Natl Acad Sci U S A. 2008;105(48):18919-18923.

45. Wilkins JA, Sansom OJ. C-Myc is a critical mediator of the phenotypes of Apc loss in the intestine. Cancer Res. 2008;68(13):4963-4966.

46. Annibali D, et al. Myc inhibition is effective against glioma and reveals a role for Myc in proficient mitosis. Nat Commun. 2014;5:4632.

47. Sulima SO, Hofman IJF, De Keersmaecker K, Dinman JD. How Ribosomes Translate Cancer. Cancer Discov. 2017;7(10):1069-1087.

48. Quin JE, Devlin JR, Cameron D, Hannan KM, Pearson RB, Hannan RD. Targeting the nucleolus for cancer intervention. Biochim Biophys Acta. 2014;1842(6):802-816.

49. Pelletier J, Thomas G, Volarević S. Ribosome biogenesis in cancer: new players and therapeutic avenues. Nat Rev Cancer. 2018;18(1):51-63.

50. Kraushar ML, et al. Thalamic WNT3 secretion spatiotemporally regulates the neocortical ribosome signature and mRNA translation to specify neocortical cell subtypes. J Neurosci. 2015;35(31):10911-10926.

51. Pfister AS, Kühl M. Of Wnts and ribosomes. Prog Mol Biol Transl Sci. 2018;153:131-155.

52. Dang CV, Reddy EP, Shokat KM, Soucek L. Drugging the 'undruggable' cancer targets. Nat Rev Cancer. 2017;17(8):502-508.

53. Grant CE, Bailey TL, Noble WS. FIMO: scanning for occurrences of a given motif. Bioinformatics. 2011;27(7):1017-1018. 